\title{
Exact and heuristic procedures for the Heijunka-flow shop scheduling problem with minimum makespan and job replicas
}

\author{
Joaquín Bautista-Valhondo ${ }^{1}$ (D)
}

Received: 23 October 2020 / Accepted: 7 June 2021 / Published online: 3 July 2021

(c) The Author(s) 2021, corrected publication 2021

\begin{abstract}
In this paper, a new problem of job sequences in a workshop is presented, taking into account non-unit demands for the jobs and whose objective is to minimize the total completion time for all the jobs $\left(C_{\max }\right)$ satisfying a set of restrictions imposed on the problem to preserve the production mix. Two procedures are proposed to solve the new problem: Mixed Integer Linear Programming and a Metaheuristic based on Multistart and Local Search. The two proposed procedures are tested using instance set Nissan-9Eng.I, in both cases giving rise to highly satisfactory performance both in quality of solutions obtained and in the CPU times required. Through a case study of the Nissan engine manufacturing plant in Barcelona, our economic-productive analysis reveals that it is possible to save an average of $€ 1162.83$ per day, manufacturing 270 engines, when we transform the current assembly line into a Heijunka-Flow Shop.
\end{abstract}

Keywords Flow shop scheduling problem $\cdot$ Overall demand $\cdot$ Heijunka $\cdot$ Mixed integer linear programming $\cdot$ Multistart . Local search · Metaheuristic

\section{Preliminaries}

The Flow Shop Scheduling Problem (FSP) is a sequencing problem that has received considerable attention from professionals and researchers in recent decades due in part to the wide range of production environments it can model [19].

A recent version of FSP is the $F m / \beta / \gamma / d_{i}$ family of sequencing problems [3] and 2020), which is to establish an application between the elements of a set $\mathrm{T}$ of ordinals ( $T$ elements) corresponding to the positions in the production sequence: $\pi(T)=\left(\pi_{1}, . . \pi_{T}\right)$, and the elements of a set $J$ of jobs or products ( $D$ elements, with $D=T$ ).

The jobs or products in group $J$ are classified into exclusive types or classes, $J_{i}$, satisfying the following properties: $J=\bigcup_{i \in I} J_{i}$ and $J_{i} \cap J_{i^{\prime}}=\emptyset, \forall\left\{i, i^{\prime}\right\} \in I$, where $I$ is the set of job types $(i=1, . ., n)$.

In $F m / \beta / \gamma / d_{i}$ problems, the $\beta$ parameter can take the permutation (prmu) or blocking (block) values, while the $\gamma$ parameter corresponds the efficiency metrics to

Joaquín Bautista-Valhondo

joaquin.bautista@upc.edu

1 IOC ETSEIB Universitat Politècnica de Catalunya, Av. Diagonal 647, 08028 Barcelona, Spain optimize $\left(C_{\max }, C_{\text {med }}\right.$, etc.), vector $\vec{d}=\left(d_{1}, d_{2}, ., d_{n}\right)$ represents the demand plan for the considered job types, and $d_{i}$ symbolizes the number of jobs of type $i \in I$ within $J$, that is to say $d_{i}=\left|J_{i}\right| \forall i \in I$, satisfying: $\sum_{\forall i} d_{i}=D=T$.

The units of $J$ travel in order through a set $K$ of $m$ stations on an assembly line arranged in series, and the production of a job of type $i \in I$ requires a heterogeneous processing time $p_{i, k}$ in workstation $k \in K(k=1, . ., m)$.

The purpose of problems $F m / \beta / \gamma / d_{i}$ is to obtain a sequence of replicated jobs or products $\left(d_{i}\right)$, in a line with $m$ machines, with the possibility of wblocking or not, according to the $\beta$ parameter, and with the objective of optimizing the efficiency metric represented by the $\gamma$ parameter $\left(C_{\max }, C_{\text {med }}\right.$, etc.).

Therefore, using the notation proposed by Graham et al. [11], both the $\mathrm{Fm} / \mathrm{prmu} / \gamma$ problems [1, 10, 13, 20, 22, 23] as the $F m / b l o c k / \gamma$ problems $[4,8,16,18,21]$ are particular cases of the family $F m / \beta / \gamma / d_{i}$, when $d_{i}=1$ for all $i \in I$.

On the other hand, completing all jobs in the shortest time possible $\left(\min C_{\max }\right)$ ) is not the only desirable objective when establishing a product manufacturing sequence. In production environments that are governed by the Just-inTime manufacturing ideals [17], the production sequences must have properties that are linked to the Heijunka concept 
$[9,12,14]$, whose meaning is to achieve regularity of production.

El The Heijunka (regularity) concept can be applied to any constituent element of Just in Time production, the most obvious criteria being the following:

C1. Regularize the consumption of the parts. The purpose of this criterion is to control the stock levels of the component parts of mixed products (e.g., in the manufacture of engines: block, cylinder head, cylinders and pistons, camshaft, gear change, etc.) throughout the manufacturing process on the assembly line.

C2. Regularize workloads at line stations. The purpose of this criterion is to avoid or smooth the work overloads that are generated when a manufacturing sequence consecutively contains a series of products rich in process time. This criterion is purely ergonomic and its objective is to avoid or reduce the risk of injury to line operators due to intermittent overloads.

C3. Regularize the manufacture of mixed products throughout the manufacturing sequence. This criterion tries to collect, in a simple way and to facilitate management, the benefits of criteria $\mathrm{C} 1$ and $\mathrm{C} 2$, since it encourages, without optimizing, both the regularity of the consumption of the component parts and the regularity of the workloads in the production line.

On the other hand, the incorporation of Heijunka in production sequence problems can be characterized by three methods:

M1. Constraints: For example, imposing minimum and maximum manufacturing levels on the job types $(i=1, \ldots, n)$ in each manufacturing cycle $(t=1, \ldots, T)$ and/or imposing minimum and maximum consumption values on the component parts of mixed products in each manufacturing cycle.

M2. Objective function: Maximizing the constancy of the product manufacturing rates [15] and/or the component consumption rates [5] and/or the rates of the required processing times in the workstations.

M3. Mixed characterization: There is also the possibility of establishing a mixed characterization of Heijunka, which incorporates into the sequence models the two previous methods: (a) restrictions and (b) an objective function.

In this work, the third criterion (C3) and the first method (M1) have been added to the genuine $F m / \mathrm{prmu} / \mathrm{C}_{\max } / d_{i}$ problem to achieve sequences with minimum makespan $\left(C_{\max }\right.$ : time that elapses from the start of work to the end) and with some properties that propitiate the regularity of product manufacturing through restrictions.
The main contributions of this work are: (i) description and formulation of a new problem that we call Hejunka $-\mathrm{Fm} / \mathrm{prmu} / \mathrm{C}_{\max } / \mathrm{d}_{i}$; (ii) design and implementation of a Metaheuristic based on Multistart and Local Search (MS-Q) to solve the new problem; (iii) a computational analysis of MS-Q and MILP (CPLEX solver) performance in CPU time and quality of solutions using real-dimension instances related to case study; and (iv) an economic-productive feasibility study to implement the solutions on a production line.

The remaining text has the following structure. Section 2 is dedicated to presenting the new problem under study which is illustrated with an example in Sect. 3. In Sect. 4, the designed MS-Q procedure is described. In Sect. 5, a case study with its data is shown, as well as the procedures used and their results. Finally, Sect. 6 offers some conclusions about this work.

\section{Heijunka $-F m / p r m u / C_{\text {max }} / d_{i}$ Problem}

To incorporate Heijunka, we will indicate that the sequence $\pi(T)=\left(\pi_{1}, \ldots, \pi_{T}\right)$, which is composed of $T$ units of jobs, has the property of preservation of the production mix if the set of restrictions (1) is satisfied. We also call this property Quota property:

$\lambda_{i} t \leq X_{i, t} \leq \lambda_{i} t \equiv\left|X_{i, t}-\lambda_{i} t\right|<1 \quad \forall i \in I, \quad \forall t \in \mathrm{T} ; X_{i, T}=d_{i} \forall i \in I$

where:

- $I$ : set of product types, $i=1, \ldots,|I|$.

- $\mathrm{T}$ : set of manufacturing cycles in every demand plan, $t=1, . .,|\mathrm{T}| ; T \equiv|\mathrm{T}|$.

- $d_{i}$ : demand for units of type $i \in I$ in an arbitrary demand plan.

- $\lambda_{i}$ : proportion of units of type $i \in I: \lambda_{i}=d_{i} / T \forall i \in I$.

- $X_{i, t}$ : number of units of type $i \in I$ in the partial sequence $\pi(t) \subseteq \pi(T)$ : actual production associated with the partial sequence $\pi(t)$.

The Quota property (1) imposes that the actual production $X_{i, t}$, for every product ( $i \in I$ and every manufacturing cycle $t \in \mathrm{T}$, must be an integer as close as possible to its ideal production $\lambda_{i} t$. The ideal production $\left(\lambda_{i} t\right)$ is defined as the quota of manufacturing time given to a product $(i \in I)$ until the end of each production cycle $(t=1, . .,|\mathrm{T}|)$.

Under such conditions, we can present a model for the $\mathrm{Fm}$ $/ p r m u / C_{\max } / d_{i}$ that accounts for two types of aspects:

Efficiency: objective function to minimize the maskespan $C_{\max }$. 
Technical-productive: Quota property to enforce preservation of the production mix in the Heijunka manufacturing sequence $\pi(T)$.

Effectively, assuming the following data is known:

- The set of job types $(I: i=1, . .,|I|)$ and the set of stations $(K: k=1, . .,|K|)$.

- The processing times $p_{i, k}(i \in I \wedge k \in K)$ of the operations.

- The demand vectors $\vec{d}=\left(d_{1}, \ldots, d_{|I|}\right)$ and production mix $\vec{\lambda}=\left(\lambda_{1}, \ldots, \lambda_{|I|}\right)$.

The problem is finding a Quota sequence of $T$ jobs $\pi(T)=\left(\pi_{1}, \ldots, \pi_{T}\right)$ with minimum makespan $C_{\text {max }}$ that satisfies the demand plan represented by the vector $\vec{d}$. The formulation of the model is as follows.

\subsection{Model Q-FSP}

$$
\begin{aligned}
& \min \mathcal{F}(\pi(T))=C_{\max } \equiv C_{m, T} \\
& C_{k, t}\left(\pi_{t}\right)=S_{k, t}\left(\pi_{t}\right)+p_{\pi_{t}, k} \forall k \in K \forall t=1, . ., T \\
& S_{k, t}\left(\pi_{t}\right)=\max \left(C_{k, t-1}\left(\pi_{t-1}\right), C_{k-1, t}\left(\pi_{t}\right)\right) \forall k \in K \forall t=1, . ., T \\
& X_{i, t}=\left|\left\{\pi_{\tau} \in \pi(t) \subseteq \pi(T): \pi_{\tau}=i \in I\right\}\right| \forall i \in I \forall t=1, . ., T \\
& \lambda_{i} t \leq X_{i, t} \leq \lambda_{i} t \forall i \in I \forall t=1, . ., T \\
& X_{i, T}=d_{i} \forall i \in I \\
& C_{k, 0}=0 \forall k \in K \\
& C_{0, t}=0 \forall t=1, . ., T
\end{aligned}
$$

In the model Q-FSP, the identity (2) expresses the minimization of the objective function $\mathcal{F}(\pi(T))$ that attends to the time of completion of the last job or product $\pi_{T}$ of the production sequence $\pi(T)$ in the last machine $(k=m)$; that is: $C_{\max } \equiv C_{m, T}$. The equality (3) determines the minimum time of completion of the $t$-th job $\pi_{t}$ in production sequence $\pi(T)$ in machine $k \in K: C_{k, t}\left(\pi_{t}\right)$. Meanwhile, the equality (4) determines the minimum start time $S_{k, t}$ of the $t$-th job $\pi_{t}$ in $\pi(T)$ in machine $k \in K$. Formula (5) serves to count the number of jobs of type $i \in I$ in the partial sequence $\pi(t) \subseteq \pi(T)$. The conditions (6) impose the Quota property on the manufacturing sequence $\pi(T)$. The equalities (7) impose the satisfaction of the demand plan $\left(d_{i} \forall i \in I\right)$. Finally, conditions (8) and (9) set the start of completion times.

\section{An illustrative example}

In order to illustrate the problem under study, the following example is presented: There are 6 jobs or products $(T=6)$, of which 3 are type A, 1 is type B, and 2 are type C. The units of product are processed in 3 workstations $(|K|=3)$ with different processing times. The processing time of each unit of type of product (A, B, C) in each workstation $\left(m_{1}, m_{2}, m_{3}\right)$ is that set out in Table 1 .

The optimal manufacturing sequence for the proposed example, in order to minimize the completion time of all the jobs on the production line $\left(C_{\max }\right)$, for the problem $\mathrm{Fm}$ $/ \mathrm{prmu} / C_{\max } / d_{i}$ is $\pi_{1}(6)=(C, C, A, A, A, B)$. Figure 1 shows the Gantt chart for this sequence.

For its part, Fig. 2 shows the Gantt chart corresponding to an optimal sequence for the problem Hejunka $-\mathrm{Fm} / \mathrm{prmu}$ $/ C_{\max } / d_{i}$, in which the satisfaction of the Quota property of all types of product is imposed in all manufacturing cycles. The sequence $\pi_{2}(6)=(C, A, A, C, A, B)$ has a value of the objective function $C_{\max }\left(\pi_{2}\right)=34$.

Considering the sequences $\pi_{1}(6)$ y $\pi_{2}(6)$, it can be stated:

(i) The solution $\pi_{1}(6)$ presents a value of $C_{\max }$ less by one unit of time than that corresponding to the solution $\pi_{2}(6)$ (i.e. : $\left.C_{\max }\left(\pi_{2}\right)-C_{\max }\left(\pi_{1}\right)=34-33=1\right)$. This means that $\pi_{1}(6)$ is more efficient than $\pi_{2}(6)$ in terms of completion time for all jobs.

(ii) The solution $\pi_{2}(6)=(C, A, A, C, A, B)$ satisfies the Quota property at all positions in the sequence.

(iii) The solution $\pi_{1}(6)=(C, C, A, A, A, B)$ violates the Quota property at 3 positions in the sequence, as detailed in Table 2.

In view of Table 2, we can state that the sequence $\pi_{1}(6)=(C, C, A, A, A, B)$ does not satisfy the Quota property for product types $\mathrm{A}$ and $\mathrm{C}$ in the cycle $t=2$ nor for product type $\mathrm{C}$ in cycle $t=3$, therefore, the sequence $\pi_{1}(6)$ violates the Quota property in $8.33 \%$ of the constraints.

In the subsection dedicated to the implementation of solutions in a production line, the advantages offered by planning sequences satisfying the Quota property within the Heijunka ideology are described.

Table 1 Processing times $\left(p_{i, k}\right)$ required by the units of product, according to type, in each workstation

\begin{tabular}{lllll}
\hline & $A\left(d_{A}=3\right)$ & $B\left(d_{B}=1\right)$ & $C\left(d_{c}=2\right)$ & $\sum_{\forall i} d_{i} \times p_{i, k}$ \\
\hline$m_{1}$ & 5 & 4 & 3 & 25 \\
$m_{2}$ & 5 & 4 & 4 & 27 \\
$m_{3}$ & 4 & 3 & 5 & 25 \\
$\sum_{\forall k} d_{i} \times p_{i, k}$ & $42(3 \times 14)$ & $11(1 \times 11)$ & $24(2 \times 12)$ & $p_{\text {tot }}=77$ \\
\hline
\end{tabular}

The total processing time required to the production line is $p_{\text {tot }}=77$ 


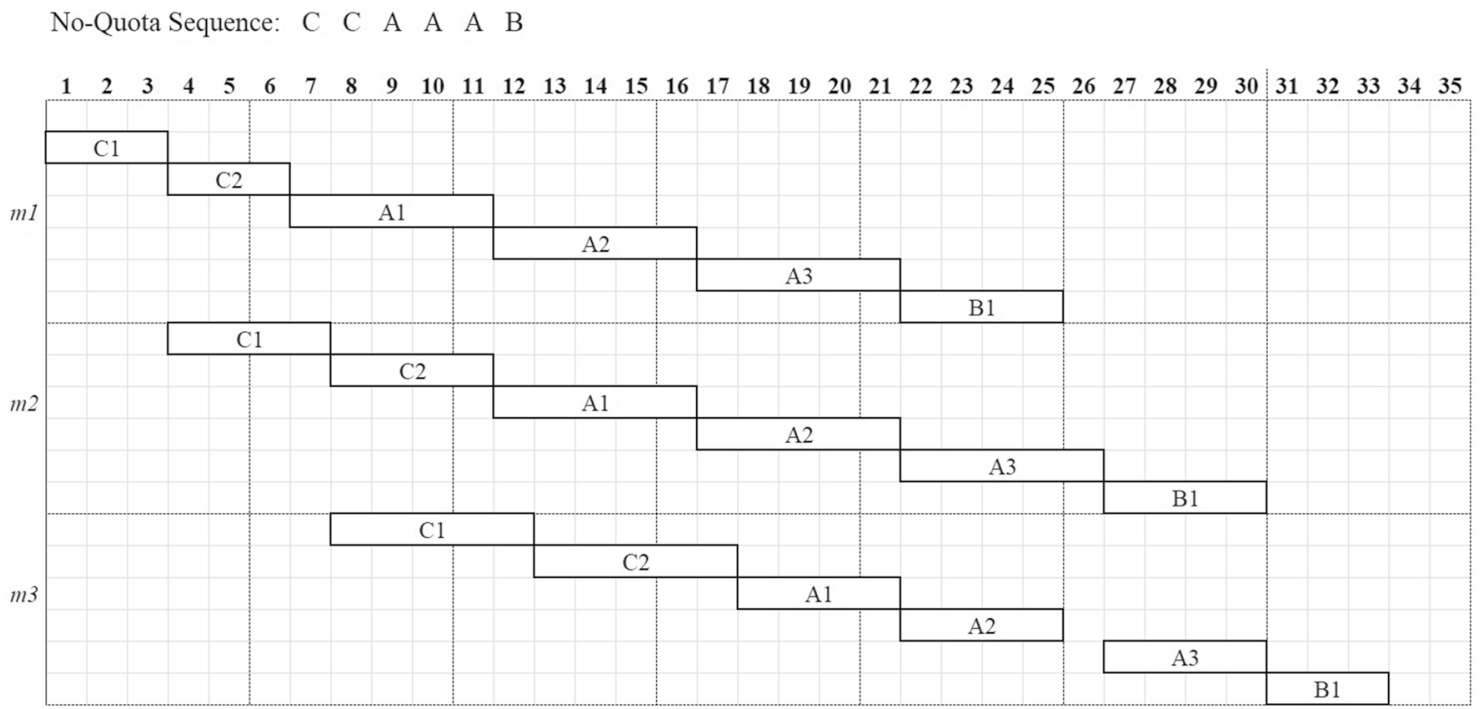

Fig. 1 Gantt chart for the sequence $\pi_{1}(6)=(C, C, A, A, A, B)$. The sequence $\pi_{1}(6)$ is optimal for the problem $F m / p r m u / C_{\max } / d_{i}$, and its value is $C_{\max }\left(\pi_{1}\right)=33$

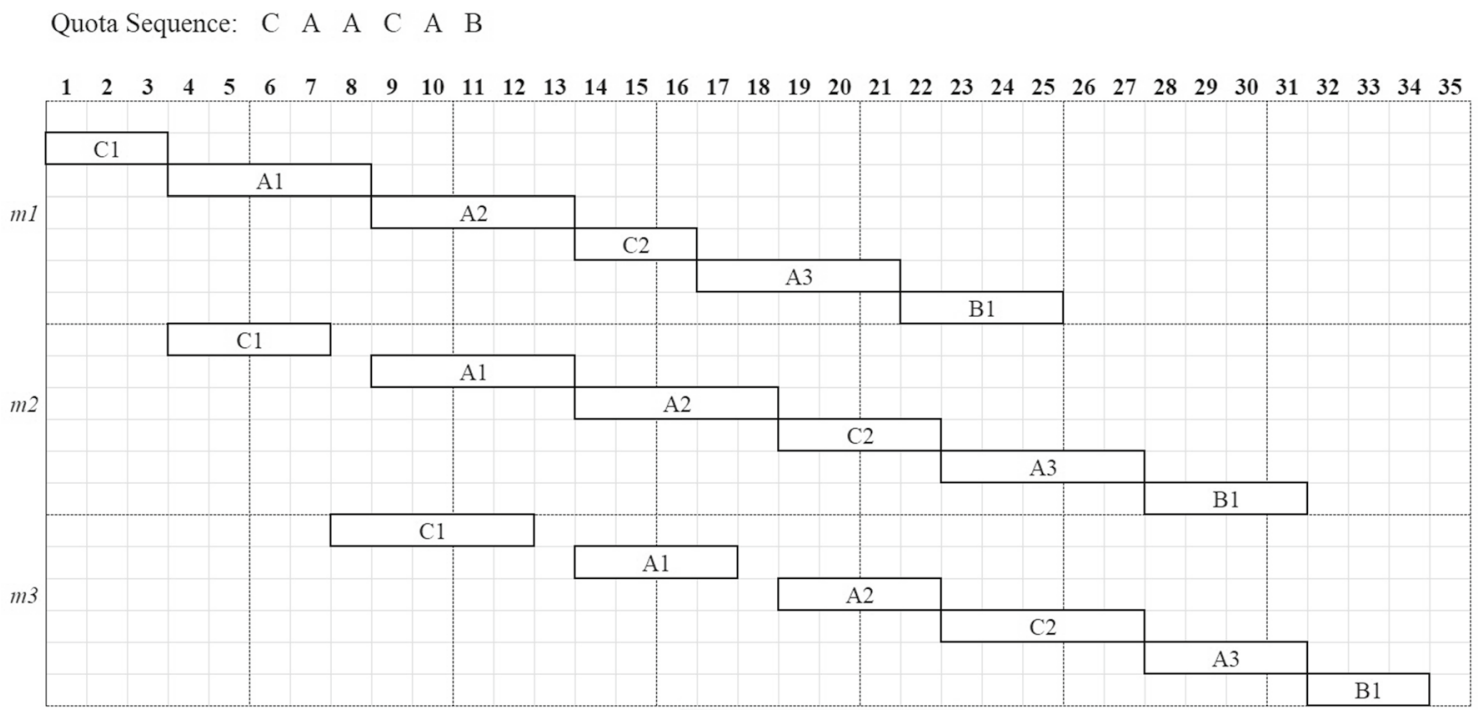

Fig. 2 Gantt chart for the sequence $\pi_{2}(6)=(C, A, A, C, A, B)$. The sequence $\pi_{2}(6)$ is optimal for the Hejunka $-F m / p r m u / C_{\text {max }} / d_{i}$ problem, and its value is $C_{\max }\left(\pi_{2}\right)=34$

Table 2 Solution $\pi_{1}(6)=(C, C, A, A, A, B)$ : the values of the accumulated productions $X_{i, t}$ and the intervals $[a, b]$ are shown

\begin{tabular}{|c|c|c|c|c|c|c|c|c|c|c|c|c|}
\hline \multirow[t]{2}{*}{$i$} & \multicolumn{2}{|c|}{$t=1$} & \multicolumn{2}{|l|}{$t=2$} & \multicolumn{2}{|l|}{$t=3$} & \multicolumn{2}{|c|}{$t=4$} & \multicolumn{2}{|c|}{$t=5$} & \multicolumn{2}{|c|}{$t=6$} \\
\hline & $X_{i, t}$ & {$[a, b]$} & $X_{i, t}$ & {$[a, b]$} & $X_{i, t}$ & $X_{i, t}$ & $X_{i, t}$ & {$[a, b]$} & $X_{i, t}$ & {$[a, b]$} & $X_{i, t}$ & {$[a, b]$} \\
\hline A & 0 & {$[0,1]$} & 0 & {$[1,1]$} & 1 & {$[1,2]$} & 2 & {$[2,2]$} & 3 & {$[2,3]$} & 3 & {$[3,3]$} \\
\hline B & 0 & {$[0,1]$} & 0 & {$[0,1]$} & 0 & {$[0,1]$} & 0 & {$[0,1]$} & 0 & {$[0,1]$} & 1 & {$[0,1]$} \\
\hline C & 1 & {$[0,1]$} & 2 & {$[0,1]$} & 2 & {$[1,1]$} & 2 & {$[1,2]$} & 2 & {$[1,2]$} & 2 & {$[2,2]$} \\
\hline
\end{tabular}

The values $a=\lambda_{i} t$ and $b=\lambda_{i} t$ are respectively lower and upper limits that are imposed on the variables $X_{i, t}(\forall i \forall t)$ to achieve a Quota sequence 


\section{Metaheuristic procedure for Heijunka - Fm/prmu/C $C_{\max } / d_{i}$}

The proposed metaheuristic is based on a Multistart procedure with Local Search similar to Bautista and Alfaro [2]. Indeed, the proposed procedure, MS-Q, consists of a first phase (constructive phase) which provides an initial solution through a randomized greedy procedure, and a second phase (improvement phase) which uses local search procedures to reach the local optima in one or more specific neighborhoods.

After setting a prefixed number of iterations (construction plus improvement), MS-Q metaheuristic obtains in phase-1 manufacturing sequences, $\pi(T)=\left(\pi_{1}, \ldots, \pi_{T}\right)$, that satisfy the Quota property, and then, in phase-2, those sequences are subjected to local optimization in order to minimize the completion time of the last job in the last workstation, that is: $C_{\max }$.

\subsection{Phase 1: construction of a Quota sequence}

The problem of the construction of a Quota sequence, which we will call Quota-Product Rate Variation Problem (Q-PRV), can be formulated as a Binary Linear Programming (BLP) representing maximum constraints satisfaction problem, as follows.

\subsection{Model maxsat Q-PRV}

$\min \mathcal{Z}_{\text {sum }}(\pi(T))=\sum_{t=1}^{T} \sum_{i=1}^{n} z_{i, t} \Leftrightarrow \max \mathcal{Z}_{\text {sum }}^{\prime}(\pi(T))=\sum_{t=1}^{T} \sum_{i=1}^{n}\left(1-z_{i, t}\right)$

$\sum_{i=1}^{n} x_{i, t}=1 \quad \forall t=1, . ., T$

$\sum_{t=1}^{T} x_{i, t}=d_{i} \quad \forall i=1, \ldots, n$

$X_{i, t}=\sum_{\tau=1}^{t} x_{i, \tau} \quad \forall i=1, . ., n ; \forall t=1, . ., T$

$\left|X_{i, t}-\lambda_{i} t\right|<1+z_{i, t} \quad \forall i=1, . ., n ; \forall t=1, . ., T$

$x_{i, t} \in\{0,1\} \quad \forall i=1, . ., n ; \forall t=1, . ., T$

$z_{i, t} \in\{0,1\} \quad \forall i=1, . ., n ; \forall t=1, . ., T$

$X_{i, t} \in \mathbb{Z}^{+} \cup\{0\} \quad \forall i=1, . ., n ; \forall t=1, . ., T$ where $x_{i, t}(\forall i \forall t)$ is a binary variable that equals 1 if and only if a unit of type of product $i \in I$ occupies position $t$ of the manufacturing sequence $\pi(T)$, while binary variable $z_{i, t}(\forall i \forall t)$ takes the value 0 when the type of product $i \in I$ satisfies the property Quota in the production cycle $t$ and is equal to 1 otherwise.

In the Maxsat Q-PRV model, the objective function (10) corresponds to the minimization of the number of Quota constraints violated $\left(\mathcal{Z}_{\text {sum }}\right)$. Equalities (11) impose that each position in the sequence has a job assigned, while equalities (12) force compliance with the demand plan $\vec{d}=\left(d_{1}, \ldots, d_{n}\right)$. The equalities (13) are used to determine the accumulated productions $X_{i, t}(\forall i \forall t)$ of all types of jobs and up to each manufacturing cycle. The inequalities (14) force the satisfaction of the Quota property by all types of jobs $(\forall i \in I)$ in all positions of the sequence $(\forall t \in \mathrm{T})$. Finally, conditions (15) and (16) impose that the variables $x_{i, t}$ and $z_{i, t}$ are binary, while conditions (17) force the accumulated production $\left(X_{i, t}\right)$ are integers and not negative.

To generate Quota sequences in accordance with the Maxsat Q-PRV model, an enumerative deterministic procedure can be designed based on the branching and cutting of partial solutions; however, in this work we have chosen to use random to promote the diversity of the initial solutions generated in Phase 1, thus allowing them to belong to different regions of the feasible solutions space.

Another indirect way of constructing sequences that satisfy all or a large part of the Quota constraints (14) is to determine integer values for the real production variables $X_{i, t}$ as close as possible to their ideal values $\lambda_{i} t$ and that, in addition, these values are consistent with the rest of the restrictions of the Maxsat Q-PRV model. To do this, it is enough to change the objective function (10) for a function that measures the discrepancies between the real and ideal accumulated productions. Some examples of discrepancy functions that we refer to are the following:

$\min \Delta_{1}(\pi(T))=\sum_{t=1}^{T} \sum_{i=1}^{n}\left(X_{i, t}-\lambda_{i} t\right)^{2}$

$\min \Delta_{2}(\pi(T))=\sum_{t=1}^{T} \sum_{i=1}^{n}\left|X_{i, t}-\lambda_{i} t\right|$

$\min \Delta_{3}(\pi(T))=\max _{1 \leq t \leq T} \max _{1 \leq i \leq n}\left(X_{i, t}-\lambda_{i} t\right)^{2}$

$\min \Delta_{4}(\pi(T))=\max _{1 \leq t \leq T} \max _{1 \leq i \leq n}\left|X_{i, t}-\lambda_{i} t\right|$

In this work, the function (18), sum of quadratic discrepancies: $\Delta_{1}(\pi(T))$, is fundamental to construct a random generator of Quota sequences. 
First, in Phase 1 a sequence of jobs $\pi(T)=\left(\pi_{1}, \ldots, \pi_{T}\right)$ is constructed satisfying the Upper Quota property ((i.e. $\left.X_{i, t} \leq \lambda_{i} t, \forall i \forall t\right)$ ), progressively and assigning at each stage $t(t=1, \ldots, T)$ a job from the $\mathrm{CL}(t)$ list of candidates that can be drawn to occupy the position $t$ of the manufacturing sequence. Consequently, when stage $t$ is reached, it is added to the sequence consolidated in the previous stage, $\pi(t-1)=\left(\pi_{1}, ., \pi_{t-1}\right)$, a job $i \in \mathrm{CL}(t)$. List $C L(t)$ is constructed like this:

$\mathrm{CL}(t)=\left\{i \in I:\left(n_{i}<d_{i}\right) \wedge\left(n_{i}+1 \leq \lambda_{i} t\right)\right\}$

where $n_{i}$ is the number of jobs of type $i \in I$ that contains the production sequence $\pi(t-1)=\left(\pi_{1}, ., \pi_{t-1}\right)$.

Therefore, for a job type $i \in I$ to enter the list $C L(t)$ of stage $t$, it must meet the following two conditions:

1. The job type does not have its demand fulfilled: $n_{i}=X_{i, t-1}<d_{i}$.

2 . The difference between the upper Quota value $\lambda_{i} t$, corresponding to the ideal production of stage $t$, and the consolidate production up to the previous stage must be greater than or equal to one unit: $\lambda_{i} t-n_{i} \geq 1$.

Note that the candidate list, $\mathrm{CL}(t)$, only contains jobs or products that satisfy the upper Quota property; this is done like this because if the strict satisfaction of the Quota property is imposed: $\lambda_{i} t \leq n_{i}+1 \leq \lambda_{i} t \equiv\left|n_{i}+1-\lambda_{i} t\right|<1$, then there is a risk, and this is often the case, that CL $(t)$ remains empty.

Second, the sum of quadratic discrepancies associated with each candidate job that is contained in the list $\mathrm{CL}(t)$ is evaluated, using the indices $g_{i}^{(t)}$ :

$g_{i}^{(t)}=\sum_{k=1}^{n}\left(n_{k}+\delta_{i, k}-\lambda_{k} t\right)^{2} \forall i \in \mathrm{CL}(t)$

where $n_{k}$ is the number of jobs of type $k \in I$ that contains the sequence consolidated in the previous stage, $\pi(t-1)$, and $\delta_{i, k}$ is the Kronecker delta: $\delta_{i, i}=1 \wedge \delta_{i, k}=0$ if $i \neq k$.
Third, the jobs in the list $\mathrm{CL}(t)$ are ordered according to the increasing order of the priority indices $g_{i}^{(t)}$, giving rise to the ordered list $\overline{\mathrm{CL}}(t)$.

Alternatively, the sorting of the list $\mathrm{CL}(t)$ to construct the list $\overline{\mathrm{CL}}(t)$ can be made more efficient by using the priority indices $f_{i}^{(t)}$ which are defined as in (24).

$f_{i}^{(t)}=\lambda_{i} t-n_{i} \forall i \in \mathrm{CL}(t)$

The equivalence between the orderings of the jobs according to the indices $g_{i}^{(t)}$ and $-f_{i}^{(t)}$ is demonstrated below.

Theorem 1 Given a partial sequence of jobs $\pi(t-1)=\left(\pi_{1}, ., \pi_{t-1}\right)$ and a list of jobs $C L(t)$ constructed according to (22), then, the ordering of jobs of CL $(t)$ according to the indices $g_{i}^{(t)}$ (see (23)) is opposite to the ordering according to the indices $f_{i}^{(t)}$ (see (24)).

Proof Indeed, let $H_{k, t}=n_{k}-\lambda_{k} t(\forall k \in I, \forall t \in \mathrm{T})$, then, it can be stated:

$g_{i}^{(t)} \leq g_{j}^{(t)} \Leftrightarrow \sum_{k=1}^{n}\left(n_{k}+\delta_{i, k}-\lambda_{k} t\right)^{2} \leq \sum_{k=1}^{n}\left(n_{k}+\delta_{j, k}-\lambda_{k} t\right)^{2} \Leftrightarrow$

$\sum_{k=1}^{n} \delta_{i, k}^{2}+\sum_{k=1}^{n} H_{k, t}^{2}+2 \sum_{k=1}^{n} \delta_{i, k} H_{k, t} \leq \sum_{k=1}^{n} \delta_{j, k}^{2}+\sum_{k=1}^{n} H_{k, t}^{2}+2 \sum_{k=1}^{n} \delta_{j, k} H_{k, t} \Leftrightarrow$

$\sum_{k=1}^{n} \delta_{i, k} H_{k, t} \leq \sum_{k=1}^{n} \delta_{j, k} H_{k, t} \Leftrightarrow H_{i, t}$

$\leq H_{j, t} \Leftrightarrow \lambda_{i} t-n_{i} \geq \lambda_{j} t-n_{j} \Leftrightarrow f_{i}^{(t)} \geq f_{j}^{(t)}$

After this ordering, the list $\overline{\mathrm{CL}}(t)$ is reduced through a mechanism that is a function of the admission factor $\alpha$ (percentage of candidate jobs), with this operation, the restricted list $\overline{\operatorname{RCL}}(t, \alpha)$ is obtained, which coincides with $\overline{\mathrm{CL}}(t)$ when $\alpha=100 \%=1$, while if $\alpha=1 /|I|$, the best candidate job from such lists is selected at each stage $t$.

Taking into account all the above, Algorithm A1 is formalized. 


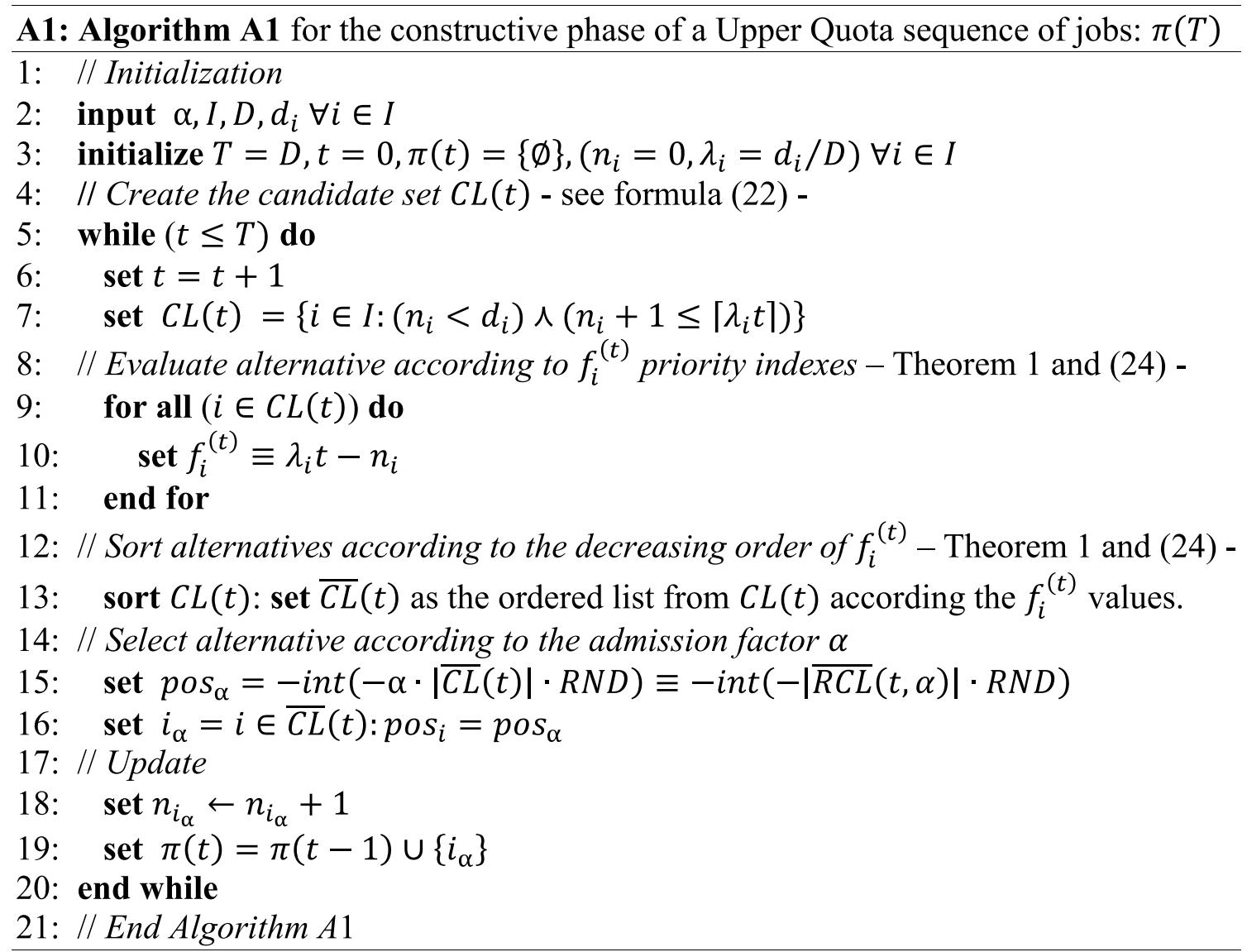

Note that Algorithm A1 is a general method of generating Upper Quota sequences, $\pi(T)$, independently of any other goal. Sometimes, the Algorithm A1 obtains solutions that also satisfy the Lower Quota property $\left(\lambda_{i} t \leq X_{i, t} \forall i \in I \forall t \in \mathrm{T}\right)$, when this purpose is not achieved then Algorithm A2 is run. 


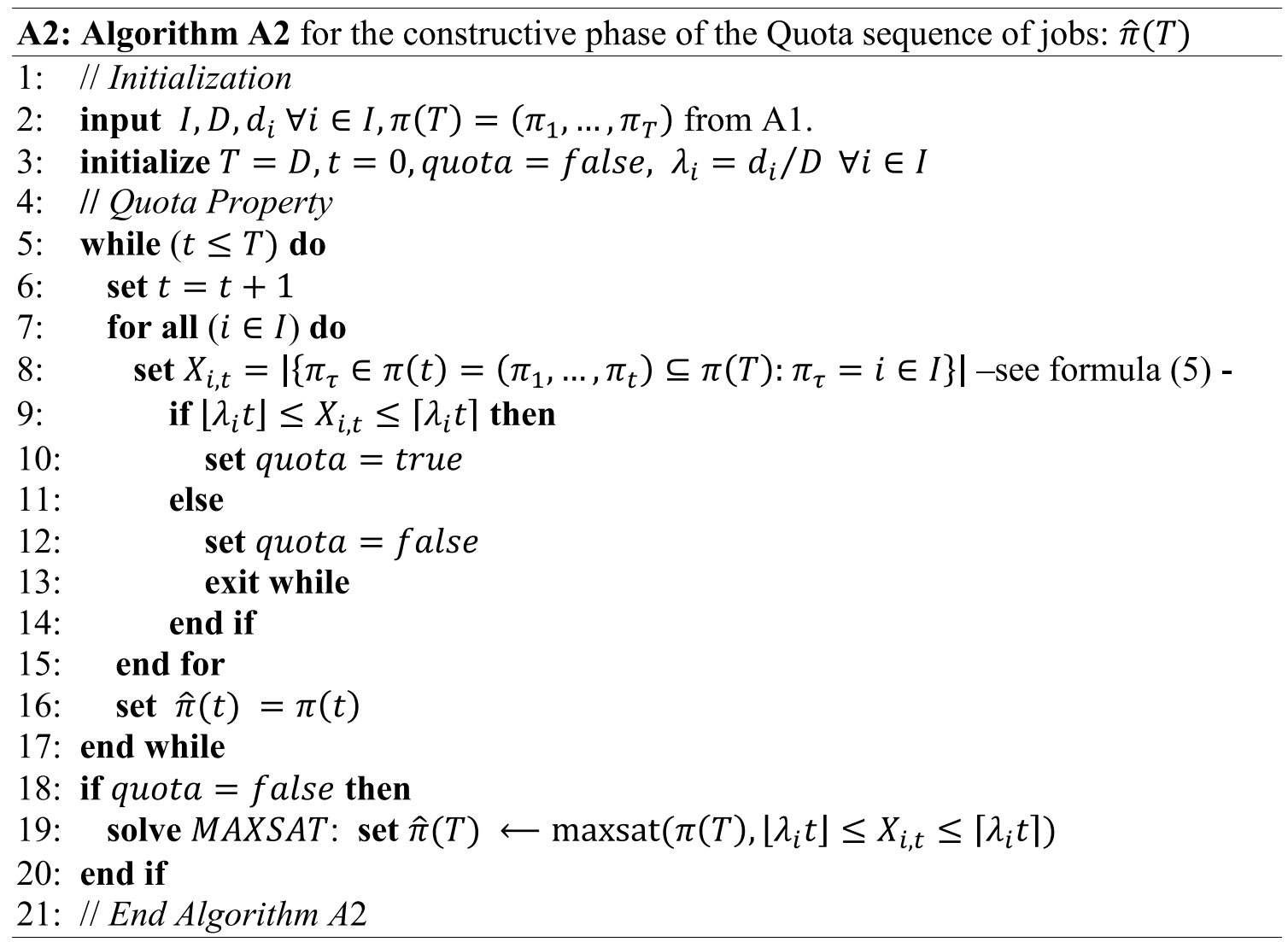

The MAXSAT procedure in A2 (Line 19 from A2) is an exchange algorithm, based on Local Search with exhaustive descent, that solves the Maxsat Q-PRV problem satisfying the constraints (14): $\left(\left|X_{i, t}-\lambda_{i} t\right|<1, \forall i \forall t\right)$, which provides as a solution a sequence $\hat{\pi}(T)$ that does satisfy the Quota property in all of the manufacturing cycles.

Specifically, MAXSAT algorithm starts from the solution $\pi(T)$ generated by Algorithm A1 and performs in each iteration the exchange of the jobs of every pair of positions of the current sequence $\hat{\pi}(T)$, consolidating, in each iteration, the Last sequence that minimizes the number of Quota constraints violated. The execution of the MAXSAT algorithm ends when $\mathcal{Z}_{\text {sum }}(\hat{\pi}(T))=0$ or $\mathcal{Z}_{\text {sum }}(\hat{\pi}(T))=|I| \times T$ (see formula (10)).

Obviously, the CPU time efficiency of the MAXSAT procedure is higher the lower the number of Quota constraints violated by the initial sequence $\pi(T)$; for this reason, the sequences provided by the A1 algorithm are used, since they comply with the Upper Quota property and tend to comply with the Lower Quota property when the values of the admission factor $\alpha$ are small.

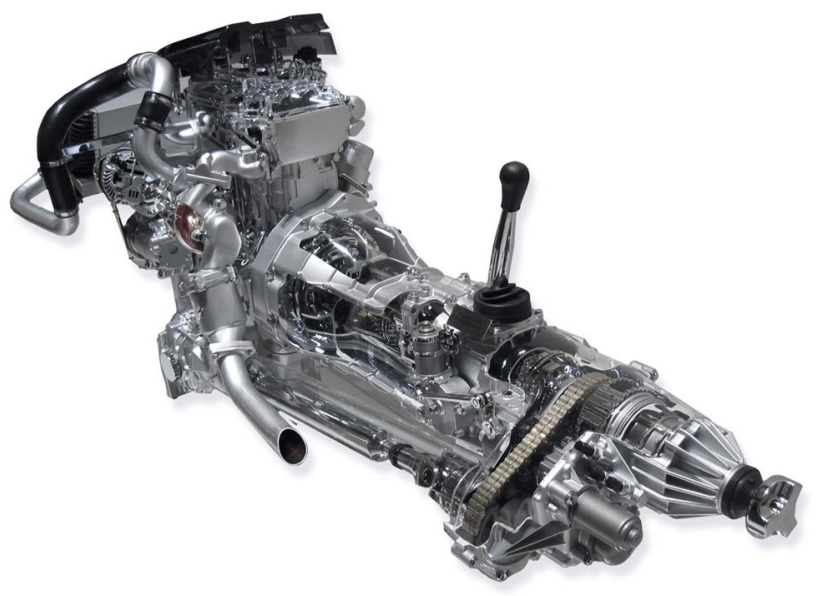

Fig. 3 Nissan Pathfinder Engine. Characteristics: (i) 747 parts and 330 references, (ii) 378 elemental assembly tasks grouped in 140 production line tasks 
Table 3 Daily demands by product type and plan $\left(d_{i, \varepsilon}\right)$ for the 23 instances Nissan-9Eng.I $(\varepsilon \in \mathrm{E})$

\begin{tabular}{llllllllllllll}
\hline$\varepsilon \in \mathrm{E}$ & 1 & 2 & 3 & 4 & 5 & 6 & 7 & 8 & 9 & SUV & Van & Truck & Total \\
\hline 1 & 30 & 30 & 30 & 30 & 30 & 30 & 30 & 30 & 30 & 90 & 60 & 120 & 270 \\
2 & 30 & 30 & 30 & 45 & 45 & 23 & 23 & 22 & 22 & 90 & 90 & 90 & 270 \\
3 & 10 & 10 & 10 & 60 & 60 & 30 & 30 & 30 & 30 & 30 & 120 & 120 & 270 \\
4 & 40 & 40 & 40 & 15 & 15 & 30 & 30 & 30 & 30 & 120 & 30 & 120 & 270 \\
5 & 40 & 40 & 40 & 60 & 60 & 8 & 8 & 7 & 7 & 120 & 120 & 30 & 270 \\
6 & 50 & 50 & 50 & 30 & 30 & 15 & 15 & 15 & 15 & 150 & 60 & 60 & 270 \\
7 & 20 & 20 & 20 & 75 & 75 & 15 & 15 & 15 & 15 & 60 & 150 & 60 & 270 \\
8 & 20 & 20 & 20 & 30 & 30 & 38 & 38 & 37 & 37 & 60 & 60 & 150 & 270 \\
9 & 70 & 70 & 70 & 15 & 15 & 8 & 8 & 7 & 7 & 210 & 30 & 30 & 270 \\
10 & 10 & 10 & 10 & 105 & 105 & 8 & 8 & 7 & 7 & 30 & 210 & 30 & 270 \\
11 & 10 & 10 & 10 & 15 & 15 & 53 & 53 & 52 & 52 & 30 & 30 & 210 & 270 \\
12 & 24 & 23 & 23 & 45 & 45 & 28 & 28 & 27 & 27 & 70 & 90 & 110 & 270 \\
13 & 37 & 37 & 36 & 35 & 35 & 23 & 23 & 22 & 22 & 110 & 70 & 90 & 270 \\
14 & 37 & 37 & 36 & 45 & 45 & 18 & 18 & 17 & 17 & 110 & 90 & 70 & 270 \\
15 & 24 & 23 & 23 & 55 & 55 & 23 & 23 & 22 & 22 & 70 & 110 & 90 & 270 \\
16 & 30 & 30 & 30 & 35 & 35 & 28 & 28 & 27 & 27 & 90 & 70 & 110 & 270 \\
17 & 30 & 30 & 30 & 55 & 55 & 18 & 18 & 17 & 17 & 90 & 110 & 70 & 270 \\
18 & 60 & 60 & 60 & 30 & 30 & 8 & 8 & 7 & 7 & 180 & 60 & 30 & 270 \\
19 & 10 & 10 & 10 & 90 & 90 & 15 & 15 & 15 & 15 & 30 & 180 & 60 & 270 \\
20 & 20 & 20 & 20 & 15 & 15 & 45 & 45 & 45 & 45 & 60 & 30 & 180 & 270 \\
21 & 60 & 60 & 60 & 15 & 15 & 15 & 15 & 15 & 15 & 180 & 30 & 60 & 270 \\
22 & 20 & 20 & 20 & 90 & 90 & 8 & 8 & 7 & 7 & 60 & 180 & 30 & 270 \\
23 & 10 & 10 & 10 & 30 & 30 & 45 & 45 & 45 & 45 & 30 & 60 & 180 & 270 \\
\hline
\end{tabular}

Table 4 Grouping of the 23 instances Nissan-9Eng.I into 7 categories of demand plans

\begin{tabular}{lll}
\hline Category & Plans & Type of demand plan \\
\hline 01 & $\# 1$ & Balanced demand for products \\
02 & $\# 2$ & Balanced demand for families \\
03 & $\# 3$ to \#5 & Very low demand for a family \\
04 & $\# 6$ to \#8 & High demand for a family \\
05 & $\# 9$ to \#11 & Very high demand for a family \\
06 & $\# 12$ to 17 & Family demand in arithmetic progression \\
07 & $\# 18$ to 23 & Family demand in hypergeometric progression \\
\hline
\end{tabular}

\subsection{Phase 2: improvement $C_{\max }$ of the quota sequences through local search}

The improvement phase starts with a Quota sequence $\hat{\pi}(T)$ in which five descent algorithms are run consecutively and repetitively in five neighborhoods (three exchange and two insertion) until none of them improves the best solution that is achieved during the iteration. From two arbitrary Quota sequences, the one that offers the least total completion time $\left(C_{\max }\right)$ is selected. The descent algorithms are based on the exchange and insertion of jobs, and they are oriented to the exploration of sequence cycles in both increasing and decreasing order. The five descent algorithms are:

LS1. Forward exchange for ranges of job types: For all $t$ position of the current sequence, $\hat{\pi}(T)$., the job type is determined that is in that position and the next closest locus is searched, $t^{\prime}>t$, that is occupied by the same type (i.e., $\hat{\pi}_{t}=\hat{\pi}_{t^{\prime}}$ ); if no such locus exists, then its value is set by making $t^{\prime}=T+1$. Just after, the tentative exchange between $\hat{\pi}_{t}$ and the jobs located in the range $\left[t+1, t^{\prime}-1\right]$ of the sequence is made. The first exchange that reduces the total completion time $C_{\max } \equiv C_{m, T}$ (see (2)) is consolidated as long as the resulting sequence satisfies the Quota property.

LS2. Backward exchange for ranges of job types: This procedure is similar to the previous one, but in this case the search is performed for $t=T$ to 1 step -1 . Obviously, if the previous closest locus, $t^{\prime}\left(t^{\prime}<t\right)$, with the same job type $\left(\hat{\pi}_{t}=\hat{\pi}_{t^{\prime}}\right)$ does not exist, it is considered $t^{\prime}=0$. The first exchange that reduces $C_{\max }$ is consolidated as long as the resulting sequence satisfies the Quota property.

LS3. Complete exchange between pairs of positions: This procedure is used to reinforce the previous two and uses a larger neighborhood. At each iteration, for all position $t$ of the current sequence $\hat{\pi}(T)$, the job 
Table 5 Processing time under normal operation $\left(p_{i, k}\right)$ in seconds of the 9 types of engines $(i \in I)$ in the 21 workstations $(k \in K)$ of the set of Nissan-9Ing.I

\begin{tabular}{lllllllllll}
\hline$k \backslash i$ & 1 & 2 & 3 & 4 & 5 & 6 & 7 & 8 & 9 & $A v$ \\
\hline 1 & 104 & 100 & 97 & 92 & 100 & 94 & 103 & 109 & 101 & 100.0 \\
2 & 103 & 103 & 105 & 107 & 101 & 108 & 106 & 102 & 110 & 105.0 \\
3 & 165 & 156 & 164 & 161 & 148 & 156 & 154 & 164 & 155 & 158.1 \\
4 & 166 & 175 & 172 & 167 & 168 & 167 & 168 & 156 & 173 & 168.0 \\
5 & 111 & 114 & 114 & 115 & 117 & 117 & 115 & 111 & 111 & 113.9 \\
6 & 126 & 121 & 122 & 124 & 127 & 130 & 120 & 121 & 134 & 125.0 \\
7 & 97 & 96 & 96 & 93 & 96 & 89 & 94 & 101 & 92 & 94.9 \\
8 & 100 & 97 & 95 & 106 & 94 & 102 & 103 & 102 & 100 & 99.9 \\
9 & 179 & 174 & 173 & 178 & 178 & 171 & 177 & 171 & 174 & 175.0 \\
10 & 178 & 172 & 172 & 177 & 178 & 177 & 175 & 173 & 175 & 175.2 \\
11 & 161 & 152 & 168 & 167 & 167 & 166 & 172 & 157 & 177 & 165.2 \\
12 & 96 & 106 & 105 & 97 & 101 & 100 & 96 & 104 & 96 & 100.1 \\
13 & 99 & 101 & 102 & 101 & 99 & 101 & 96 & 102 & 99 & 100.0 \\
14 & 147 & 155 & 142 & 154 & 146 & 143 & 154 & 153 & 155 & 149.9 \\
15 & 163 & 152 & 156 & 152 & 153 & 152 & 154 & 156 & 156 & 154.9 \\
16 & 163 & 185 & 183 & 178 & 169 & 173 & 172 & 182 & 171 & 175.1 \\
17 & 173 & 179 & 178 & 169 & 173 & 178 & 174 & 175 & 175 & 174.9 \\
18 & 176 & 167 & 181 & 180 & 172 & 173 & 173 & 168 & 184 & 174.9 \\
19 & 162 & 150 & 152 & 152 & 160 & 151 & 155 & 148 & 167 & 155.2 \\
20 & 164 & 161 & 157 & 159 & 162 & 160 & 162 & 158 & 157 & 160.0 \\
21 & 177 & 161 & 154 & 168 & 172 & 170 & 167 & 149 & 169 & 165.2 \\
\hline & & & & & & & & & &
\end{tabular}

of the locus $t$ is exchanged with the job of the locus $t^{\prime} \in[t+1, T]$, if $\hat{\pi}_{t} \neq \hat{\pi}_{t^{\prime}}$. The last job exchange that minimizes $C_{\max } \equiv C_{m, T}$ is consolidated, provided the Quota property is satisfied.

LS4. Forward insertion for ranges of job types: For all $t$ position of the current sequence, $\hat{\pi}(T)$, the job type in the $t$ position is detected and the next closest locus $t^{\prime}\left(t^{\prime}>t\right)$ is searched that is occupied by the same type $\left(\hat{\pi}_{t}=\hat{\pi}_{t^{\prime}}\right)$; if these locus does not exist, it is considered $t^{\prime}=T+1$. Following, the $\hat{\pi}_{t}$ job is inserted in the range of sequence positions $\left[t+1, t^{\prime}-1\right]$. Then, the first insertion that leads to reduce $C_{\max } \equiv C_{m, T}$ is done as long as the resulting sequence satisfies the Quota property.

LS5. Backward insertion for ranges of job types: This insertion procedure is similar to LS4 with respect to the neighborhood, and analogous in the search for types of jobs to LS2.

While there is improvement, the above five algorithms are repeated.

\section{A case study in an engine plant}

\subsection{Data set}

The computational experience proposed here is focused on comparing the MS-Q and MILP (Mixed Integer Linear Programming) procedures in terms of the quality of the solutions and the CPU times. As in Bautista-Valhondo and Alfaro-Pozo [7], the analysis is related to a case study of the Nissan plant in Barcelona: an assembly line of nine types of engines grouped into three families: SUVs, Vans and Trucks (see an engine example in Fig. 3). The production line under study employs 42 operators work in shifts of $8 \mathrm{~h}$, and the significant data of this case are the following:

- There are 9 job types $(|I|=9)$ so that each job type corresponds to a type of engine.

- The workshop (line) has 21 workstations $(|K|=21$ ) arranged in series.

- In this work, we consider 23 engine demand plans $|\mathrm{E}|=23$ (see Table 3 ).

- The daily demand is 270 jobs for all demand plans $T \equiv D_{\varepsilon}=270$ jobs $(\forall \varepsilon \in \mathrm{E})$.

- The demand plans have been grouped into 7 categories (see Table 4).

- The values of the processing times at normal work pace $p_{i, k}(\forall i \in I, \forall k \in K)$ are between 89 s and 185 s (see Table 5). 
Table 6 Results for $C_{\max }$ (seconds) and Gap (in millionths) for Nissan-9Eng.I instances using MILP-1, MILP-2 and MS-Q

\begin{tabular}{|c|c|c|c|c|c|c|c|c|c|}
\hline \multirow[t]{2}{*}{$\varepsilon \in \mathrm{E}$} & \multicolumn{2}{|l|}{ MILP-1 } & \multicolumn{4}{|l|}{ MILP-2 } & \multicolumn{3}{|l|}{ MS-Q } \\
\hline & $C_{\max }^{1}$ & $\mathrm{CPU}$ & $L B$ & $C_{\max }^{2}$ & Gap & $\mathrm{CPU}$ & $C_{\max }^{3}$ & Gap & $\mathrm{CPU}$ \\
\hline 1 & 50,091 & 45.8 & 50,100 & 50,101 & 20 & 3600.6 & 50,101 & 20 & 176.8 \\
\hline 2 & 50,174 & 15.2 & 50,180 & 50,180 & 0 & 366.7 & 50,180 & 0 & 130.8 \\
\hline 3 & 50,301 & 10.3 & 50,303 & 50,303 & 0 & 37.9 & 50,303 & 0 & 15.5 \\
\hline 4 & 50,167 & 13.6 & 50,170 & 50,170 & 0 & 38.6 & 50,170 & 0 & 213.0 \\
\hline 5 & 50,379 & 9.9 & 50,385 & 50,385 & 0 & 45.7 & 50,385 & 0 & 73.6 \\
\hline 6 & 50,202 & 14.3 & 50,202 & 50,202 & 0 & 14.1 & 50,204 & 40 & 2.9 \\
\hline 7 & 50,395 & 8.3 & 50,397 & 50,397 & 0 & 33.4 & 50,397 & 0 & 180.1 \\
\hline 8 & 50,123 & 12.4 & 50,126 & 50,128 & 40 & 3600.3 & 50,130 & 80 & 233.5 \\
\hline 9 & 50,378 & 10.4 & 50,378 & 50,378 & 0 & 17.0 & 50,378 & 0 & 5.3 \\
\hline 10 & 50,619 & 7.6 & 50,625 & 50,625 & 0 & 9.0 & 50,625 & 0 & 15.9 \\
\hline 11 & 50,078 & 25.3 & 50,084 & 50,084 & 0 & 162.4 & 50,086 & 40 & 48.7 \\
\hline 12 & 50,192 & 17.4 & 50,196 & 50,196 & 0 & 102.4 & 50,196 & 0 & 176.0 \\
\hline 13 & 50,123 & 14.8 & 50,126 & 50,136 & 199 & 3600.3 & 50,136 & 199 & 12.7 \\
\hline 14 & 50,218 & 10.1 & 50,223 & 50,223 & 0 & 134.7 & 50,224 & 20 & 48.7 \\
\hline 15 & 50,242 & 10.5 & 50,242 & 50,242 & 0 & 105.0 & 50,242 & 0 & 175.7 \\
\hline 16 & 50,118 & 55.8 & 50,123 & 50,123 & 0 & 160.3 & 50,128 & 100 & 129.0 \\
\hline 17 & 50,269 & 10.6 & 50,273 & 50,273 & 0 & 74.0 & 50,275 & 40 & 4.3 \\
\hline 18 & 50,273 & 14.3 & 50,273 & 50,273 & 0 & 15.1 & 50,275 & 40 & 8.3 \\
\hline 19 & 50,475 & 8.1 & 50,481 & 50,481 & 0 & 7.8 & 50,481 & 0 & 15.0 \\
\hline 20 & 50,089 & 96.1 & 50,100 & 50,100 & 0 & 65.2 & 50,100 & 0 & 48.1 \\
\hline 21 & 50,307 & 13.8 & 50,307 & 50,307 & 0 & 10.5 & 50,307 & 0 & 5.4 \\
\hline 22 & 50,539 & 7.3 & 50,545 & 50,545 & 0 & 9.3 & 50,545 & 0 & 31.9 \\
\hline 23 & 50,151 & 11.0 & 50,157 & 50,157 & 0 & 44.0 & 50,158 & 20 & 24.3 \\
\hline$A v$ & $50,256.7$ & 19.3 & $50,260.7$ & $50,261.3$ & 11.3 & 532.8 & $50,262.0$ & 26.0 & 77.2 \\
\hline $\operatorname{Max}$ & 50,619 & 96.1 & 50,625 & 50,625 & 199 & 3600.6 & 50,625 & 199 & 233.5 \\
\hline Min & 50,078 & 7.3 & 50,084 & 50,084 & 0 & 7.8 & 50,086 & 0 & 2.9 \\
\hline
\end{tabular}

Columns $C P U$ show the CPU time (seconds) spent solving each instance
All the production plans shown in Table 1 have been used to carry out the computational experimentation developed in this work. As said, the total number of engines assembled in a working day is 270 in two shifts. The 7 categories that allow the grouping of demand plans are summarized in Table 4.

Meanwhile, the values of the processing times $p_{i, k}(\forall i \in I, \forall k \in K)$ for each job type and for each workstation are shown in Table 5.

\subsection{Procedures and computational analysis}

The compiled codes of the procedures that we have selected in this work are MILP (1 and 2) and MS-Q (running in Intel(R) Core (TM) i7-8750H CPU @ $2.21 \mathrm{GHz}, 16 \mathrm{~GB}$ RAM, $\times 64$ Windows 10 Pro). Table 6 shows the best results with respect to $C_{\max }$ and CPU Time from MILP (1 and 2) and MS-Q procedures for the 23 datasets of the problem $\varepsilon \in \mathrm{E}$.
In "Appendix I", the 46 best Quota-sequences obtained by MILP-2 and MS-Q are published.

In Table 6, the column headings represent the following characteristics:

$\varepsilon \in \mathrm{E}$ Identification number of the instances for Plan\#1 to Plan\#23

$C_{\max }^{1} \quad$ Optimal value of makespan for the $\mathrm{Fm} / \mathrm{prmu} / \mathrm{C}_{\max } / d_{i}$ problem obtained for MILP-1

$C_{\max }^{2} \quad$ Best makespan value for the Heijunka $-\mathrm{Fm} / \mathrm{prmu} / \mathrm{C}_{\max } / d_{i}$ problem obtained for procedure MILP-2

$C_{\max }^{3} \quad$ Best makespan value for the Heijunka $-\mathrm{Fm} / \mathrm{prmu} / C_{\max } / d_{i}$ problem obtained for procedure MS-Q

$L B \quad C_{\max }$ lower limit for the Heijunka $-F m /$ block $/ C_{\max } / d_{i}$ problem obtained for MILP-1 or MILP-2 using the CPLEX solver

Gap Relative gap between $C_{\max }^{h}(h \in\{2,3\})$ and $L B$ measured in millionths

The relative gap values (measured in millionths) between $C_{\max }^{k}$ and $L B$ is calculated using formula (25). 
Table 7 Some properties of the performance of MS-Q with the set of instances Nissan9Engine-I

\begin{tabular}{|c|c|c|c|c|c|c|c|}
\hline \multirow[t]{2}{*}{$\overline{\varepsilon \in \mathrm{E}}$} & \multicolumn{3}{|c|}{ Phase 1} & \multicolumn{4}{|l|}{ Phase 2} \\
\hline & $\alpha^{*}(\varepsilon)$ & $r_{m x s}^{*}(\varepsilon)$ & $\% r_{\text {no } \_}(\varepsilon)$ & $\operatorname{iter}^{*}(\varepsilon)$ & $\operatorname{sol}^{*}(\varepsilon)$ & $n \_\operatorname{Sol}(\varepsilon)$ & $\mathrm{CPU}_{1}(\varepsilon)$ \\
\hline 1 & 0.50 & 0 & 0 & 13 & 114 & 171 & 13.28 \\
\hline 2 & 0.33 & 0.83 & 0.171 & 12 & 90 & 161 & 11.64 \\
\hline 3 & 0.11 & 1.00 & 6.638 & 1 & 2 & 2 & 15.50 \\
\hline 4 & 1.00 & 0.89 & 4.432 & 9 & 97 & 206 & 23.08 \\
\hline 5 & 0.50 & 1.00 & 2.858 & 7 & 48 & 142 & 10.54 \\
\hline 6 & 0.11 & 0 & 0 & 1 & 2 & 2 & 2.86 \\
\hline 7 & 0.33 & 0.94 & 0.597 & 16 & 123 & 159 & 11.46 \\
\hline 8 & 0.33 & 0.94 & 0.362 & 20 & 156 & 156 & 11.68 \\
\hline 9 & 0.11 & 1.00 & 0.006 & 1 & 2 & 2 & 5.28 \\
\hline 10 & 1.00 & 1.00 & 12.442 & 2 & 8 & 129 & 11.94 \\
\hline 11 & 0.50 & 1.00 & 2.372 & 3 & 36 & 195 & 14.83 \\
\hline 12 & 1.00 & 0.90 & 6.798 & 10 & 75 & 161 & 18.74 \\
\hline 13 & 0.11 & 0 & 0 & 1 & 8 & 8 & 12.69 \\
\hline 14 & 0.50 & 0.75 & 1.409 & 4 & 32 & 164 & 12.88 \\
\hline 15 & 0.50 & 0.90 & 1.488 & 10 & 128 & 285 & 18.31 \\
\hline 16 & 0.20 & 0 & 0 & 12 & 115 & 176 & 10.04 \\
\hline 17 & 0.11 & 0 & 0 & 1 & 2 & 2 & 4.34 \\
\hline 18 & 0.11 & 1.00 & 0.796 & 1 & 3 & 3 & 8.30 \\
\hline 19 & 0.33 & 1.00 & 1.529 & 2 & 9 & 128 & 9.07 \\
\hline 20 & 0.33 & 1.00 & 0.434 & 3 & 30 & 208 & 16.93 \\
\hline 21 & 0.11 & 1.00 & 0.541 & 1 & 3 & 3 & 5.39 \\
\hline 22 & 0.33 & 1.00 & 1.996 & 4 & 20 & 109 & 8.83 \\
\hline 23 & 0.33 & 1.00 & 0.422 & 2 & 15 & 250 & 14.87 \\
\hline Average & - & 0.75 & 1.969 & 6 & 49 & 123 & 11.85 \\
\hline Maximum & - & 1.00 & 12.442 & 20 & 156 & 285 & 23.08 \\
\hline Minimum & - & 0 & 0 & 1 & 2 & 2 & 2.86 \\
\hline
\end{tabular}

$G a p(h, \varepsilon)=10^{6} \times \frac{C_{\max }^{h}(\varepsilon)-L B(\varepsilon)}{L B(\varepsilon)} \forall h \in\{2,3\}, \forall \varepsilon \in \mathrm{E}$

The characteristics of the procedures are:

- MILP-1: Model $F m / p r m u / C_{\text {max }} / d_{i}$ : (i) Objective function for minimizing the $C_{\max }$ value of the production sequence; (ii) implementation for IBM ILOG CPLEX solver (Optimization Studio v.12.2, win- $\times 86-64)$; (iii) maximum CPU time of $180 \mathrm{~s}$ allowed for solving each instance ( 23 instances). The average CPU time used by each demand plan to find the optimal solution is equal to $19.3 \mathrm{~s}$. This procedure is used to determine adjusted lower bounds for the problem under study.

- MILP-2: Model Hejunka - Fm/prmu/C $C_{\max } / d_{i}$ (this work): (i) Objective function for minimizing the $C_{\max }$ value of the Quota production sequence; (ii) implementation for IBM ILOG CPLEX solver (Optimization Studio v.12.2, win- $\times 86-64)$; (iii) maximum CPU time of $3600 \mathrm{~s}$ allowed for solving each instance (23 instances). The average CPU time used by each demand plan to find the best solution is equal to $532.8 \mathrm{~s}$.

- MS-Q: Is the Multistart algorithm presented in this work, which is focused on minimizing the total completion time $C_{\max }$ in Quota manufacturing sequences. The maximum number of iterations for each demand plan from Nissan-9Eng.I instances is equal to 20 with five candidate admission factors $\alpha=(0.11,0.20,0.33,0.50,1)$, which generates in the constructive phase 1863 solutions and 14,110 improved solutions (improvement phase) in 115 executions. MS-Q uses on average a CPU time equal to $77.2 \mathrm{~s}$ to find the best solution for each demand plan and each admission factor $\alpha$.

On the other hand, an analysis of Table 6 reveals the following:

- Procedure MILP-1 obtains and ensures optimal solutions in all instances with 270 jobs (23 instances Nissan9Eng.I) when the $F m / p r m u / C_{\max } / d_{i}$ problem is solved (see column $C_{\max }^{1}$ in Table 6 ). The solutions obtained by MILP-1 do not necessarily satisfy the Quota property: 
MILP-1 violates the Quota property in 18 of 23 demand plans.

- Procedure MILP-2 obtains and ensures optimal solutions in 20 of the 23 instances with 270 jobs when the Heijunka $-\mathrm{Fm} / \mathrm{prmu} / \mathrm{C}_{\max } / d_{i}$ problem is solved (see column $C_{\max }^{2}$ in Table 6). All the solutions obtained by MILP-2 satisfy the Quota property.

- Procedure MS-Q obtains optimal solutions in 13 of the 23 instances with 270 jobs when the Heijunka-Fm $/ p r m u / C_{\max } / d_{i}$ problem is solved (see column $C_{\max }^{3}$ in Table 6). All the solutions obtained by MS-Q satisfy the Quota property.

- Regarding the value of objective $C_{\max }$, on average, MS-Q solutions differ by $0.7 \mathrm{~s}$ from MILP-2, in a range of values between 0 and $5 \mathrm{~s}$ (see columns $C_{\max }^{2}$ and $C_{\max }^{3}$ in Table 6), when considering a 50,770 s workday to build 270 engines. Consequently, MS-Q solutions can be considered equivalent to MILP-2 from the perspective of the management of productive operations.

- The average value of the relative gap between $C_{\max }^{2}$ and $L B$ achieved by MILP-2 is $1.13 \mathrm{E}-05$ in a range of values between 0 and 1.99E-04.
- The average value of the relative gap between $C_{\text {max }}^{3}$ and $L B$ achieved by MS-Q is $2.60 \mathrm{E}-05$ in a range of values between 0 and 1.99E-04.

- The average CPU times used by MILP-1 (to determine lower bounds for the problem under study) are approximately $19.3 \mathrm{~s}$ for each instance of 270 jobs in a range of values between 7.3 and $96.1 \mathrm{~s}$, when a maximum CPU time equal to $180 \mathrm{~s}$ is imposed on CPLEX to solve each instance for $\mathrm{Fm} / \mathrm{prmu} / \mathrm{C}_{\max } / d_{i}$ problem.

- The average CPU times used by MILP-2 are approximately $532.8 \mathrm{~s}$ for each instance of 270 jobs in a range of values between 7.8 and $3600.6 \mathrm{~s}$, when a maximum CPU time equal to $3600 \mathrm{~s}$ is imposed on CPLEX to solve each instance of the problem under study.

- The average CPU time used by MS-Q is equal to $77.2 \mathrm{~s}$ within a range of values between 2.9 and $233.5 \mathrm{~s}$, when 20 iterations are performed with the algorithm.

- In average CPU times, MS-Q is 6.902 times faster than MILP-2.
Table 8 Results corresponding to the savings in euros $G(\cdot)$ and the increase in engine production $\Delta P(\cdot)$ for Nissan9Eng.I instances using procedures MILP-1, MILP-2 and MS-Q

\begin{tabular}{|c|c|c|c|c|c|c|}
\hline \multirow[t]{2}{*}{$\varepsilon \in \mathrm{E}$} & \multicolumn{2}{|l|}{ MILP-1 } & \multicolumn{2}{|l|}{ MILP-2 } & \multicolumn{2}{|l|}{ MS-Q } \\
\hline & $G(1, \varepsilon)$ & $\Delta P(1, \varepsilon)$ & $G(2, \varepsilon)$ & $\Delta P(2, \varepsilon)$ & $G(3, \varepsilon)$ & $\Delta P(3, \varepsilon)$ \\
\hline 1 & 1552.00 & 3.88 & 1529.14 & 3.82 & 1529.14 & 3.82 \\
\hline 2 & 1362.29 & 3.41 & 1348.57 & 3.37 & 1348.57 & 3.37 \\
\hline 3 & 1072.00 & 2.68 & 1067.43 & 2.67 & 1067.43 & 2.67 \\
\hline 4 & 1378.29 & 3.45 & 1371.43 & 3.43 & 1371.43 & 3.43 \\
\hline 5 & 893.71 & 2.23 & 880.00 & 2.20 & 880.00 & 2.20 \\
\hline 6 & 1298.29 & 3.25 & 1298.29 & 3.25 & 1293.71 & 3.23 \\
\hline 7 & 857.14 & 2.14 & 852.57 & 2.13 & 852.57 & 2.13 \\
\hline 8 & 1478.86 & 3.70 & 1467.43 & 3.67 & 1462.86 & 3.66 \\
\hline 9 & 896.00 & 2.24 & 896.00 & 2.24 & 896.00 & 2.24 \\
\hline 10 & 345.14 & 0.86 & 331.43 & 0.83 & 331.43 & 0.83 \\
\hline 11 & 1581.71 & 3.95 & 1568.00 & 3.92 & 1563.43 & 3.91 \\
\hline 12 & 1321.14 & 3.30 & 1312.00 & 3.28 & 1312.00 & 3.28 \\
\hline 13 & 1478.86 & 3.70 & 1449.14 & 3.62 & 1449.14 & 3.62 \\
\hline 14 & 1261.71 & 3.15 & 1250.29 & 3.13 & 1248.00 & 3.12 \\
\hline 15 & 1206.86 & 3.02 & 1206.86 & 3.02 & 1206.86 & 3.02 \\
\hline 16 & 1490.29 & 3.73 & 1478.86 & 3.70 & 1467.43 & 3.67 \\
\hline 17 & 1145.14 & 2.86 & 1136.00 & 2.84 & 1131.43 & 2.83 \\
\hline 18 & 1136.00 & 2.84 & 1136.00 & 2.84 & 1131.43 & 2.83 \\
\hline 19 & 674.29 & 1.69 & 660.57 & 1.65 & 660.57 & 1.65 \\
\hline 20 & 1556.57 & 3.89 & 1531.43 & 3.83 & 1531.43 & 3.83 \\
\hline 21 & 1058.29 & 2.65 & 1058.29 & 2.65 & 1058.29 & 2.65 \\
\hline 22 & 528.00 & 1.32 & 514.29 & 1.29 & 514.29 & 1.29 \\
\hline 23 & 1414.86 & 3.54 & 1401.14 & 3.50 & 1398.86 & 3.50 \\
\hline Average & 1173.37 & 2.93 & 1162.83 & 2.91 & 1161.14 & 2.90 \\
\hline Maximum & 1581.71 & 3.95 & 1568.00 & 3.92 & 1563.43 & 3.91 \\
\hline Minimum & 345.14 & 0.86 & 331.43 & 0.83 & 331.43 & 0.83 \\
\hline
\end{tabular}


- In average relative gap, MILP-2 solutions are at 1.13E-05 of the lower bound while MS-Q solutions are at 2.60E-05 of that bound, which constitutes a technical tie.

For its part, Table 7 shows some properties on the performance of the MS-Q procedure, both in its construction phase and in its improvement phase, when the set of Nissan-9Eng.I instances is solved.

In Table 7, the column headings represent the following characteristics:

\begin{tabular}{|c|c|}
\hline$\varepsilon \in \mathrm{E}$ & $\begin{array}{l}\text { Identification number of the instances for Plan\#1 to } \\
\text { Plan\#23 }\end{array}$ \\
\hline$\alpha^{*}(\varepsilon)$ & $\begin{array}{l}\text { Best admission factor in } \mathrm{A} 1 \text {, } \\
\quad \alpha \in\{0.11,0.20,0.33,0.50,1\} \text {, for each } \varepsilon \in \mathrm{E}\end{array}$ \\
\hline$r_{m x s}^{*}(\varepsilon)$ & $\begin{array}{l}\text { Utilization rate of MAXSAT procedure in } \mathrm{A} 2 \text { for the best } \\
\text { solutions of each demand plan } \varepsilon \in \mathrm{E}\end{array}$ \\
\hline$r_{n o \_}(\varepsilon)$ & $\begin{array}{l}\text { Rate dissatisfaction of the Quota constraints (from A1) for } \\
\text { the best solutions of each demand plan } \varepsilon \in \mathrm{E} \text {. It is meas- } \\
\text { ured as a percentage: } \% r_{n o}(\varepsilon) \text {. The maximum number } \\
\text { of Quota constraints is: }|I| \times T \equiv|I| \times D\end{array}$ \\
\hline $\operatorname{iter}^{*}(\varepsilon)$ & $\begin{array}{l}\text { Iteration corresponding to the best solution of each } \\
\text { demand plan } \varepsilon \in \mathrm{E}\end{array}$ \\
\hline $\operatorname{sol}^{*}(\varepsilon)$ & $\begin{array}{l}\text { Number of solutions improved by Local Search (BL1 to } \\
\text { BL5) to get the best solution locally optimal of each } \\
\text { demand plan } \varepsilon \in \mathrm{E}\end{array}$ \\
\hline$n \_\operatorname{Sol}(\varepsilon)$ & $\begin{array}{l}\text { Number of solutions improved by Local Search (BL1 to } \\
\text { BL5) limiting the MS-Q procedure to a maximum } 20 \\
\text { iterations, for each demand plan } \varepsilon \in \mathrm{E}\end{array}$ \\
\hline
\end{tabular}

\begin{tabular}{ll}
\hline$\varepsilon \in \mathrm{E}$ & $\begin{array}{l}\text { Identification number of the instances for Plan\#1 to } \\
\text { Plan\#23 }\end{array}$ \\
\hline$C P U_{1}(\varepsilon)$ & $\begin{array}{l}\text { CPU time (seconds) per iteration, limiting the MS-Q } \\
\text { procedure to a maximum } 20 \text { iterations, for each demand } \\
\text { plan } \varepsilon \in \mathrm{E}\end{array}$
\end{tabular}

\subsection{Economic-productive feasibility study}

In this subsection, we carry out an analysis of the results considering two aspects: economic and productive.

The first aspect aims to evaluate the economic savings in euros that result from transforming the original assembly line with a cycle time $c=175 s$ into a regular flow workshop in the context of the $\mathrm{Fm} / \mathrm{prmu} / \mathrm{C}_{\max } / d_{i}$ problem.

The second aspect of the productive type is intended to measure the drop in engine production generated by the use of Heijunka concept, used in Just in Time production systems, when imposed on manufacturing sequences that satisfy the Quota property; in this case, we will use the Heijunka - Fm/prmu/C $C_{\max } / d_{i}$ model.

To carry out this analysis, the following hypotheses are taken into account:

h1. The current engine assembly line is made up of 21 workstations arranged in series. At each workstation, a team consisting of two operators operates ( 42 operators in total).

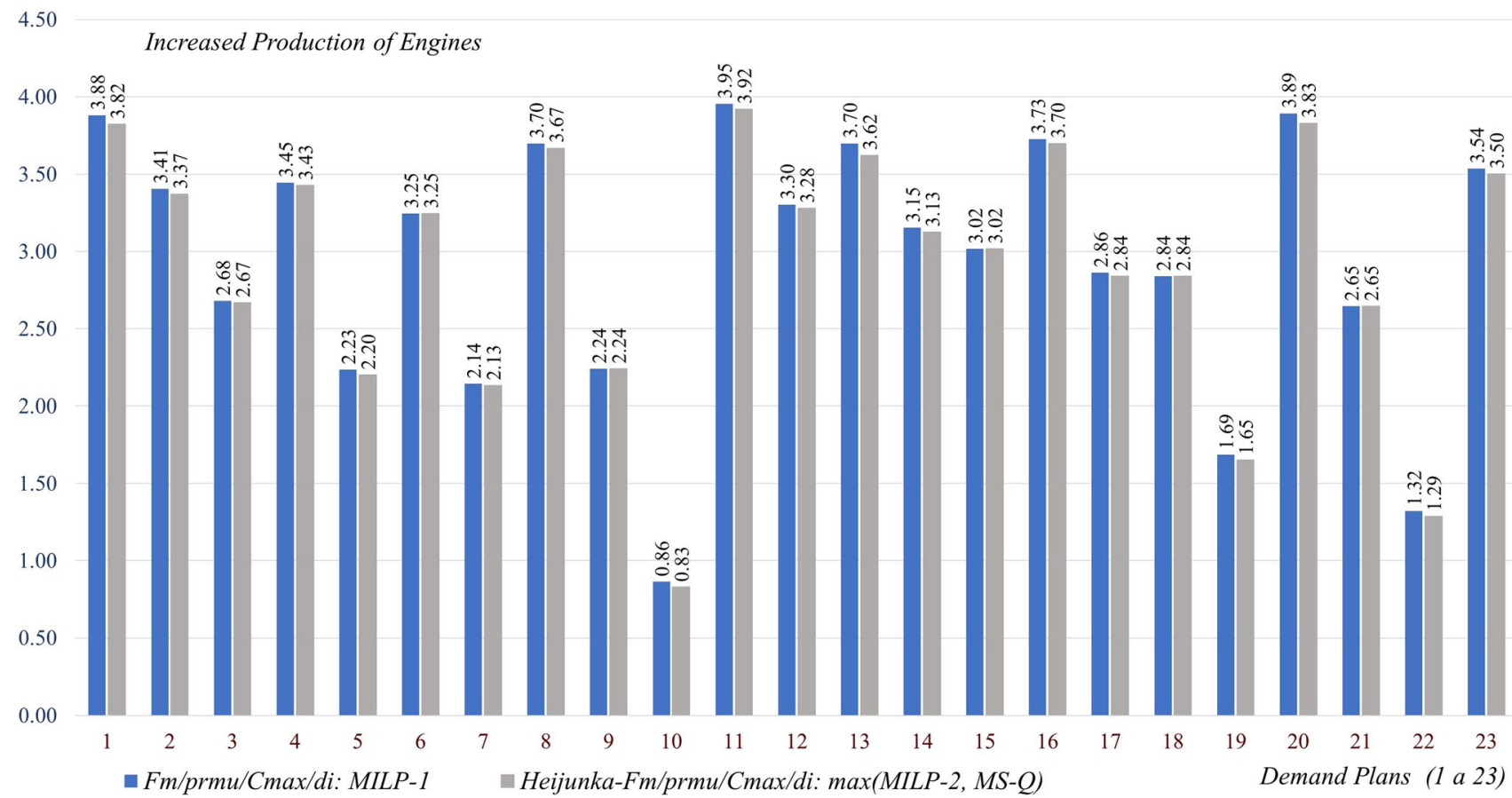

Fig. 4 Daily increased in engine production, over that of the current assembly line, obtained with procedures MILP-1 and MILP-2 or MS-Q for the Nissan-9Eng.I instance set 
h2. The current assembly line has a daily production capacity equal to 270 engines. Each production day is divided into two work shifts and each work shift has a productive time equal to $7.05 \mathrm{~h}$, after subtracting the scheduled rest times during the work day, the full duration of which is equal to $8 \mathrm{~h}$ per shift.

h3. The cost of loss engine production [6] has been valued at $\varphi=2.28757$ euros per productive second. The cost $\varphi$ is calculated taking into account three factors: (i) the average value of a motor that is equal to $€ 4,000$, (ii) the value added to the product by the assembly line that is equal to $10 \%$ of the value of the motor, and (iii) the cycle time of the line that is equal to $175 \mathrm{~s}$, that is, $c=175 \mathrm{~s}$ and the temporary window is $l_{k}=175 \mathrm{~s}$.

h4. Assuming a cycle time $c=175 s$ and that the assembly line is made up of 21 stations arranged in series, the manufacture of the 270 engines requires a time equal to $C_{\max }^{0}=50770$ seconds to complete the 270 jobs when there is no work in progress on the line (noWIP). Therefore, the direct benefit provided by the line is equivalent to $€ 108,000$ per day.

Under these conditions, the daily savings in euros $G(\cdot)$ and the daily increases in the production of $\Delta P(\cdot)$ motors, achieved with the transformation of the current assembly line into a regular flow workshop, are shown in Table 8.

In Table 8 , the $G(\cdot)$ and $\Delta P(\cdot)$ values are determined according to (26) and (27).

$G(h, \varepsilon)=\varphi \times\left(C_{\max }^{0}-C_{\max }^{h}(\varepsilon)\right) \forall h \in\{1,2,3\}, \forall \varepsilon \in \mathrm{E}$

$\Delta P(h, \varepsilon)=\frac{C_{\max }^{0}-C_{\max }^{h}(\varepsilon)}{c} \forall h \in\{1,2,3\}, \forall \varepsilon \in \mathrm{E}$

The analysis of Table 8 allows to obtain the following conclusions:

- The daily saving in euros, $G(1, \varepsilon)$, achieved with the transformation of the current line in a flow shop $\mathrm{Fm}$ $/ p r m u / C_{\max } / d_{i}$, manufacturing 270 engines per day, is equal to $€ 1173.37$ on average. Such savings are included in the interval [345.14, 1581.71], and their values depend on the demand plan used $(\varepsilon \in \mathrm{E})$.

- In case of having the same time to produce as the current one (i.e., $C_{\max }^{0}=50770 \mathrm{~s}$ ). , the estimate of the average daily increase in engine production is $\Delta P(1, \varepsilon)=2.93$, by transforming the line into a flow shop and assuming that the demand plans $(\varepsilon \in \mathrm{E})$ do no vary. These increases are included in the interval [0.86, 3.95], and their values depend on the demand plan used (see Fig. 4).

- The transformation of the current line into a regular flow shop subject to Heijunka concept (Heijunka - Fm/prmu $/ C_{\text {max }} / d_{i}$ ) leads to a maximum average saving equal to
$€ 1162.83$ per day (see average maximum between the columns $G(2, \varepsilon)$ and $G(3, \varepsilon)$ in Table 8) when 270 engines are manufactured per day. In this case, said savings are included in the interval $[331.43,1568.00]$ and their values depend on the demand plan used.

- In the case of the Heijunka-flow shop, the estimate of the daily increase in engine production with respect to the current line is equal to 2.91 engines on average (see average maximum between columns $\Delta P(2, \varepsilon)$ and $\Delta P(3, \varepsilon)$ in Table 8), provided that the original production mix does not vary in the demand plans. Here, these increases (engines per day) are included in the interval $[0.83,3.92]$ (see Fig. 4).

Figure 4 reveals similar performance between the two types of flow shops analyzed, with respect to increased productivity on the assembly line.

In fact, for all demand plans, MILP-1 solutions (18 of which do not satisfy the Quota property) correspond to increases in productivity, since the values of $\Delta P(1, \varepsilon)$ are all positive. Taking into account that the average daily increase is equal to $\Delta P(1)=2.93$ engines, it turns out that the average increase in productivity is $1.09 \%$ when the current assembly line becomes a regular flow shop $\left(\mathrm{Fm} / \mathrm{prmu} / \mathrm{C}_{\max } / d_{i}\right)$.

For its part, if it is imposed in the previous flow shop that also conforms to some requests of the Heijunka concept (Heijunka $-\mathrm{Fm} / \mathrm{prmu} / \mathrm{C}_{\max } / d_{i}$ ), it also turns out that all the solutions obtained with MILP-2 and MS-Q correspond to positive values $\Delta P(2, \varepsilon)$ y $\Delta P(3, \varepsilon)$ for all demand plans. Therefore, the Heijunka-flow shop also promotes an increase in productivity with respect to the current assembly line, with an average increase in the order of $1.08 \%$, considering the value $\Delta P(2)=2.91$ engines per day corresponding to MILP-2, or $\Delta P(3)=2.90$ engines per day corresponding to MS-Q.

Note that the solutions offered by MS-Q and MILP-2 (see Tables 6 and 8) are equivalent from a technical-productive point of view, since on average the difference between their respective times required to manufacture a total of 270 engines is equal to 0.7 s (i.e., $50,262.0-50,261.3$ ) using sequences that satisfy the Quota property, this value is negligible compared to the current available time $\left(C_{\max }^{0}=50770 \mathrm{~s}\right)$ to manufacture 270 engines, which corresponds to a working day with just over 14 operating hours equally distributed between two work shifts.

\subsection{Advantages and disadvantages of using MILP and MS- $Q$ procedures}

The solutions offered by MILP-2 and MS-Q, for the set of Nissan-9Eng.I instances, can be considered technically equivalent in terms of the value of $C_{\max }$ (see "Appendix I"); therefore, we can conclude that both procedures are equally 
valid to solve the problem Hejunka - Fm/prmu/C $C_{\max } / d_{i}$. However, since these procedures are of a different nature, it is necessary to highlight some advantages and disadvantages in relation to the application of each of them.

- In the specialized literature, most articles on the $\mathrm{Fm}$ $/ p r m u / C_{\max }$ use heuristic and metaheuristic methods. The new problem proposed in this paper is more complex than the $\mathrm{Fm} / \mathrm{prmu} / \mathrm{C}_{\max }$, so there is no reason to rule out the use of heuristics to solve the Hejunka - Fm/prmu $/ C_{\max } / d_{i}$ problem (Laplace's principle of insufficient reason). The use of Mixed Integer Linear Programming (MILP) for flow shop problems is less widespread for both reference instances and realistic cases.

- MILP-2 uses the IBM CPLEX solver, which is commercial software that requires a license in its professional version. CPLEX incorporates the most efficient optimization techniques related to MILP, and its efficiency is widely recognized in the scientific field, requiring knowledge of modeling techniques. In contrast, MQ-S is simple and easy to implement.

- MILP-2 is an application based on an exact procedure (MILP), while MS-Q is an approximation algorithm.

- MS-Q is on average about 7 times faster than MILP-2 for the Nissan-9Eng.I instance set with 270 jobs and 21 machines.

- MS-Q operates on the set of feasible solutions, and the CPU time to converge to a local optimum is predictable based on neighborhoods. For its part, the MILP technique, as a branch and bound procedure, operates with non-feasible solutions (non-integer solutions) and, therefore, the CPU time used to reach a local (or global) optimum is much less predictable. In fact, in this work, the standard deviation of the CPU time spent by MILP-2 is $\sigma_{M I L P-2}^{C P U}=1190.63$, while for MS-Q it is $\sigma_{M S-Q}^{\mathrm{CPU}}=77.24$.

\subsection{Implementation of solutions in a production line}

Taking into account the previous results, our proposal is to transform the current assembly line, with fixed cycle time and closed stations, into a regular flow workshop with open workstations within the framework of the Heijunka concept; this proposal is based on two evidences: one of an economic nature and the other of an organizational and management nature.

The first evidence is the possibility of saving an average of $€ 1162.83$ per day by manufacturing 270 engines of various types or, alternatively, the possibility of producing on average 272.91 engines per day (instead of 270) while maintaining the current working hours (14.103 h).
The second evidence is in the organizational advantages for the management offered by the level production both in the plans and in the sequences of mixed models.

The level production concept is inherent in Heijunka's ideology, and we have applied it here by enforcing the conformity of the Quota property with manufacturing sequences. Among the productive and administrative advantages offered by Heijunka are the following:

(1) Reduction of the stock level of the types of engines and engine components (Parts).

(2) Adjustment of production capacity to the demand for engines.

(3) Reduction of delivery times in all phases of the production system.

(4) Reduction of the volume of information to direct the operations of the production system.

(5) Ability to react to fluctuations in demand, since the preservation of the production mix means keeping the manufacturing system at its center of gravity from the production-demand point of view.

Having seen the advantages that a Heijunka-flow shop offers compared to a mixed model assembly line from a productive point of view, it is worth asking how to implement a solution $\left(\pi(T)=\left(\pi_{1}, ., \pi_{T}\right)\right)$ when the virtual barrier of setting the manufacturing rate by cycle time $c$ is removed.

This seemingly harmless fact involves converting current workstations to open stations, leading to a release such that both the start and end of jobs on each workstation do not occur periodically according to the value of the cycle time $c$ (v.gr. $175 \mathrm{~s}$ ), but they occur at irregular intervals that will depend on the duration of each job and the times of completion of the jobs in the current station and in the previous one.

To implement a $\pi(T)$ solution in the workshop, it is necessary that at least the following conditions are met:

c1. The manufacturing sequence must comply with the standards established in the collective agreement between the employee and the company. Compliance with this condition is guaranteed because all processing times (see Table 5) have been calculated at normal work pace and the productive time to manufacturing 270 engines ( $14.103 \mathrm{~h}$ using two shifts) takes into account the scheduled rest and forced stop times within the law.

c2. Workshop operators must be kept informed about the rhythm and the progress of production at their workstations: every operator should know the following data at the all times: (i) the engine type that reaches your workstation; (ii) the subset of tasks that makes up the job in progress; (iii) the start instant of the job in progress; (iv); the processing time required to complete 
the job in progress at normal work pace; and (v) the time available to carry out the job in progress.

Condition c2 can be easily achieved using technologies of Internet of Things (IoT) within the framework of Industry 4.0 , implementing an information system assisted by wireless connection between the central computer from production management and a set of customized tablets (42 tablets to cover the 21 workstations).

In this way, the set of tablets will visually and acoustically report on production progress at all times and on all workstations. Consequently, all operators will automatically receive the following personalized signals:

1. Audible and visual warning that indicates the beginning of a job.

2. Accelerated audible and visual warning when the time available to complete a job is ending.

3. Visual warning of the dynamic list of pending tasks on a job with the possibility that operator validates the concluded tasks and actualizes the list of tasks.

Updating activities are possible in our case, since a job is made up of 6 tasks on average and the processing times of the jobs are between 89 and $185 \mathrm{~s}$ (see Table 5), these times are sufficiently large to update the information in each workstation.

\section{Conclusions}

In this work, a new manufacturing sequence model is presented which incorporates some Heijunka properties from Just-in-Time into the $F m / p r m u / C_{\text {max }} / d_{i}$ problem. This extension (Heijunka $-\mathrm{Fm} / \mathrm{prmu} / \mathrm{C}_{\max } / d_{i}$ ) arises from our concern to adapt academic problems to problems closest to industrial reality in the automotive sector.

The dimension of the mathematical model corresponding to the problem presented depends on the number of types of jobs, the number of workstations and the total demand for products (engines) in a sequencing horizon. For example, the
MILP formulation requires at least 13,770 variables (2430 of them binary) and 25,682 constraints, for 9 types of jobs, 21 workstations and 270 products to be manufactured.

Two methods have been used to solve the new problem applied to a case study based on an engine assembly line. The first of them is based on Mixed Integer Linear Programming, and the CPLEX solver has been used solving all 23 realistic instances from the Nissan-9Eng.I set. The second method, with which the same instances have been solved, is a multistart procedure in whose constructive phase initial solutions are generated satisfying the Quota property, while in the second phase the solutions are improved using five neighborhood (three exchange and two insertion) and attending to the criterion of minimum total completion time $\left(C_{\max }\right)$.

Both procedures have been highly competitive with the new problem, since they have been able to optimally solve a high percentage of the instances using reasonable CPU times. Specifically, procedure MILP-2 obtains and ensures optimal solutions in 20 of the 23 instances with 270 engines using an average CPU time equal to $532.8 \mathrm{~s}$ for each instance with an average value of the relative gap between $C_{\max }$ and the best lower bound equal to 11.3 millionths. For its part, MS-Q has been able to obtain 13 optimum within 23 instances using an average CPU time equal to $77.2 \mathrm{~s}$ for each instance with an average Gap equal to 26.0 millionths. Therefore, it can concluded that both procedures are valid to solve the Heijunka $-\mathrm{Fm} / \mathrm{prmu} / \mathrm{C}_{\max } / d_{i}$ problem with a dimension adjusted to the automotive industry. However, although the solutions offered by MILP-2 and MS-Q can be considered equivalent in terms of the value of the objective function, it can be stated that MS-Q beats MILP computationally, being 6.902 times faster in CPU time in the experimental framework of the present case study.

Regarding the transformation of the current assembly line into a Heijunka-flow shop, the economic-productive feasibility study reveals that it is possible to save an average of $€$ 1162.83 per day by manufacturing 270 engines or, alternatively, that it is possible to produce 3 more engines per day with the current working hours.

Finally, for future lines of work, we propose to incorporate in the presented model other productive concepts such as the activity factor of the operators and the possibility of blocking the productive flow between the workstations, as well as the incorporation of some desirable properties in the workloads of the manufacturing sequence. 


\section{Appendix I: Best sequences \\ for Heijunka-Fm/prmu/C $C_{\text {max }} / d_{i}$ with the set \\ of instances Nissan-9Eng.l}

Best Quota sequences from MILP-2. Headers (Plan\#n, Cmax, Lower Bound, CPU Time in seconds)

PLAN\#1Q 50101501003600.586

581423679789513642781632495871269453189726345327184956 937815246218763495275318964827135946817294365318792456 317258649128967354983274561238741956317298546853974621 259834176236845791127358964279385164813297546296437851 436972815739126485735849126397684125374912685913457628

PLAN\#2Q 5018050180366.68

521849734656527143539824871154635294129534876254137854 365964721845135264395492781456321854375426197945451236 828145753496521834347652195843574621395194726524453819 273654541812587364395412576384941295764835521349761245 358491276854312458749653124539781254463759412135524768

PLAN\#3Q 503035030337.851

526847549758453649785644159478519546287545469857446593 589147546847529456785645943578941654785452946843759546 284574569738456954184756954876454951387454569872545694 584756914875429654987544365578461954547852946893547654 572849546978454365984571456358746495249857456491554768

PLAN\#4Q 501705017038.569

526391278934716281361238795891327164623851739972136824 987123516378622491593816723178932146793528162391728364 521839167673221894573193286871936234512718639792168324 157693218873924163253719286174962318237518963291723648 187396215279318246673183592671932814569233781962471328

PLAN\#5Q 503855038545.693

512845324413515349723455124546324153542812594315345214 134525346745125274531342154128543145524632541543129543 531452421835412534754256341625344151359245143282543541 123457745512439534521243451253145864153422543915341542 135452342756413245513245461245314351249537425413512548

PLAN\#6Q 502025020214.106

284531236123974125834136521273412359813452613412329571 235814369217213425538143621213743952124583912635173214 825731243614923125836112354475321239124713526531389124 471232561325389412593113724382162534614592313452173128 524361129538314723519217324654123138721539624513214328

PLAN\#7Q 503975039733.399

524937545458114465384525497542564451854532645443951547 584452349651454357725458149455645241583413549456524547 543149584552415467384525644543524597845512435449156547 354418955445216457945835246454352547185414545254964537 384551246455945247965445341358544527965413544512454578 
PLAN\#8Q 50128501263600.312

563972884167913845297678459661784952897635284146797835 613978456972895264173846957162963845987728546971389564 127496385978617245689758346369784592791846725168795483 631978564276983254796845791368456279487913566298784159 639476725898416375789624915273568496478916375891257468

PLAN\#9Q 503785037816.981

283123134123512291433172123632123115321735231212931234 123138526312137214321321532132219431523132316231721234 512331289123361214323512321731263214312832153211321324 523114321232139162345231312138213232171536291321321324 134215323127123831421263312312123513251432193212731328

PLAN\#10Q 50625506259.044

545844515456459445544325457544554745545845345425445541 544565449255474545354545481545445435514545244654559445 345645452545495454454515447545465425548544545345451594 534547455645425454545148454355442545545474546545451549 543454255458445754545645415344525454495545474515454548

PLAN\#11Q 5008450084162.42

563897781968967894678672997856723864919678987678967854 629739687895689467976879861678259478967638597896489761 738697289678566948976874197626958879786496739678968185 724967739856869798798674168967856297967386597897869841 736789689672469589784761896379576896784976891876975628

PLAN\#12Q 5019650196102.445

591864725394613845279845725463184752165974983154625984 761354827594567438169354248759316245785634912584715634 979854467252591864735184346259745219684351857642795694 173854126845379254196854354263797485135864796524185346 729584541293764985132546977845135648479125369645125748

PLAN\#13Q 50136501263600.349

524387196123587412965334918245413627795851263432518412 953674139852647123147852963526413852174973523841295614 326845917234351895764126312854976312453827549132514836 792134152879364556312425478139612387514634295241379625 412859873612451733986524137249518365124743915624125738

PLAN\#14Q 5022350223134.718

523849516214753842143523951467283456151294327458731954 523614125841363524927453186954351247124358132654975436 122845153147523649583214791452263458915437624152133845 527412659431485937213654234158917542361254378514293524 162547354312469531287495124365431852479135624135125478

PLAN\#15Q 5024250242105.037

561845239476254457148953589146742553943264175481583524 647557498952614534152467983154746853524173945645842591 463251784524395856454791234875649521453764852157349854 654713495462951827543451683546754299543612475851424953 548145763129456875244935584174639512547849635415254768 
PLAN\#16Q 5012350123160.264

562847913381754952631824597634219876545789123436142895 647352971285647318369452741638547291583126975341289654 237846541927586413379256184357968142532759641349785218 467356291296734581239854571268449317325469817352465891 236475891345629781345724961237584691735624189531752468

PLAN\#17Q 502735027374.047

523847514694253841535926415437584921135456427358445219 325467541289354516447612583945531247215438454659715342 365849214517645243589215473453542198645247351612545834 725453174653984215451436249285957341563454127935645412 341254887594352641355472961254384514357965241315245478

PLAN\#18Q 502735027315.122

238121345623123145253134127234123915238131524274311235 356212431312932154324121835534213721623114523531221634 315429312573213214523112394315224137253136124852311324 521334218751324132156231324912315324235114326751324132 513239124512313824351216324314923512152371324513213428

PLAN\#19Q 50481504817.811

524854354954457654584145459354564475457845254456415594 485549543245564547854145954456547541584457546254954435 154854945456543547584425594654543457954845564542451547 584345549645754452945845154564435547654457549245854145 543854564524475549584645415354954457245645945574154548

PLAN\#20Q 501005010065.203

526897789631738964795628718629769841789631623987758694 967281738695497816287369684597789263759816684279739186 378196782946935786785926417896981672789326781596347896 495786138796821769473698729682187569489376571896789632 457896781396927816785496723896781693487296517896967328

PLAN\#21Q 503075030710.545

238612341723913521523183721623913124581232931312617234 523113782931623124521731823132912634573128132316921324 571236132123913824573124312931623218571231932632181324 753216312231913824562311327123912834153217362912323184 417326123183921325523197312416323812196321423153721328

PLAN\#22Q 50545505459.294

524854574453415534524495541254534645451854594453524541 524453546754154452547541453548543154524415549546425435 654534485542415254534145457534564452541594543425415549 543475465245154354425514854452345549154745645254345514 435452854415435245574456514354524594451534524475514548

PLAN\#23Q 501575015743.954

592876784961876549769834857169789456397856784596978624 581796794826784596756849783956879146279568784936789546 547896297568873946785964817936789546876149586749987256 687539964874789265746859189763879546289764576849985761 736849725986746958784965917386497586649587918746975628 
Best Quota-sequences from MS-Q. Headers (Plan\#n, Cmax, Lower Bound, CPU Time in seconds)

PLAN\#1Q 5010150100176.758

562891347652934817823764195321487569418579263278459136 795861432295386417536487192294873165139678542296317458 861425739632984175943512687538164729856321974418762539 876132954954213768935742618938124675983456127849172365 349652781346129875934187652953721468931247568913547628

PLAN\#2Q 5018050180130.824

547216843259654831529347851493417256524936154378561428 754912739465842153584173924562536714835427919456342851 325148647517693254941235785846315294753614245698152834 574972361453295184574631246853219457835794152624934815 736549125834641275384195624537489152476359125364125748

PLAN\#3Q 503035030315.496

546834975861455497754968542458945367756425984659457184 685454379814597465564952748184575496978456452487543956 748524695386544759645751489256445897745639485489546157 736549845265487945891544657279544568453794856614557894 745245869347584695458174956357496854784952456495157468

PLAN\#4Q 5017050170212.965

526398137891267341273152689639124378971522681731389624 672193831269571384293615827689732141693722318752138649 692817351792314386972625831417936238197268213573861942 781936231865427192631273958312987146695328273168721943 614973218392615782733986412691852373912615784923176328

PLAN\#5Q 503855038573.626

541234517534621543423154852125453341294534175942152543 142654353128451254473531452348152451356429354152245431 214534253164951425352347514215745314243652854313545421 314255462851342495143251453724354151482356254413155423 345274152934152541533145429542345162534132154453152748

PLAN\#6Q 50204502022.855

523812413523469713862141235214373915296835124213724315 376412352158249312317248153429631513286324159321145372 265137324112483952813231645715231943291683425712331425 123851243451237619239143852415237162391763425812331425 231592437125864311325419632124357138923514271254316328

PLAN\#7Q 5039750397180.110

534895445712453654245175458463459452815435454647529145 459715454283451645354254745846594513524438547545695214 458521445374954625415473546554184395854224545694751543 458351447556245493541249548554612754534548647534259145 548569414545327453954581424535645247945136454524514578 
PLAN\#8Q 5013050126233.543

576913894267857461259873964851263748976489785641359278 369529746684917258985174673895676894324615273586981749 176348957596264887367929415516874289358467197863259486 796324597718546318429678695397815764852973461297688495 745362178996734589652647818937469685412735796819756248

PLAN\#9Q 50378503785.281

231281363512431239123412321521327133213214321532183216 321732153219123143214321325321763213211234312532713281 321321954213932163211232153721342132133512381234216231 321392153216423123152312831421321321353712432131262371 321532114923238131213526132312379124321512313231241328

PLAN\#10Q 506255062515.883

544654541545345745455425454954485465547454534545124545 454954541584545425455465453454954415542545437545445475 544584545254615445345549544554754524545844541534564545 549544573454515454245456548545454453145454542584594545 745546544514545425455439454545145435254654544554574548

PLAN\#11Q 500865008448.682

589637794867916828769984769367689517889677896785296894 736279468976786957889469167628597783966858779916486876 798972859668497791866398765397768874698627959788661794 936788695297868764199778496268975768976489586877369971 368796896874928675746981896375698779687491869687957628

PLAN\#12Q 5019650196175.992

572684354961319542877645598214596374812564459837578423 196145752649581437638954752946235481648527961745145393 825846577394512864561994852741354687369415824759746295 431852453767819425639458175284669574533412648579417526 895439413857526849316145274895534678941257346915256748

PLAN\#13Q 501365012612.691

523714651392874129563184342651297583124675823945147312 345968359142712568631425834172935496127623138548715249 548137342679128543625614329791528314573268549132741635 512894645837123671392451246728513994256338174255413962 871395245137624813579426131842546931258471395621245738

PLAN\#14Q 502245022348.688

561374529148342925365114853247496715232435518134945223 561417354862957314225634481815957234542311463925527634 814925471253543716283145259874145136428352374125439815 126645542137948253941365714285453312914652274853619254 135734512894456321537249125346531487129354652413125748

PLAN\#15Q 5024250242175.659

564957418324615273548496548513542974781562453436594152 478525698434157491654753952484785139546253146547249865 123545784164259358947417562415534395248469751563748425 841359456297544618753412554396587494528176425318452456 975434192581457349654351267548493581452479635512454768 
PLAN\#16Q 5012850123129.008

579834621417865523932484519766127853496128354793729514 216748539245867138915264453239876741856453219739815462 213549766284583917167453824375691952642835179462418357 298673451289345417532694517836741298539645218361729458 563477291362584541896372416538497129563871249153547628

PLAN\#17Q 50275502734.336

583427541456391295426584315743546712542318945356442815 354274591681754432524679513454213554829571483526451347 654935421518439542752134569347521848546519432561453924 735485214416537482543215497654315254829563714195342457 584163425396547241553814452963548127549316245315245748

PLAN\#18Q 50275502738.297

524316231348213251124933125734213152241323591523143218 371421352325612134523412361234151327413823125124313259 145233281154237312142536312154239312142537312142539312 146233512142537312142536312812435312423123615142538312 374123512194235312932412531317523241321453621154321328

PLAN\#19Q 504815048115.000

594458574254645345514845945456451475745425654845594345 745546854145345549458456254459354547425514745645594845 458465754354245945845745145546415945954645854745534245 854475456354145945254456457459514485245453945854754645 584547645345245945451548654345945754459645145745524548

PLAN\#20Q 501005010048.132

526897739864916287186397968735976824916782793681947586 269783491876758936796285178639896274897164839167975268 784962618793578629136978496587723896768913719486679825 674598127698632798178639568179376894958726874196632987 957368721698814796297836487961873659894726891576967328

PLAN\#21Q 50307503075.385

283123914231635721823412391321732156631213824175329312 312812395164327312823123516431273129823123516431273129 823123516431273129823123516431273129312812395164327312 312912385167324312312912358167233142312912386371425312 295132341182631273731293621241531823591231324712631328

PLAN\#22Q 505455054531.922

514453459245854451524435475534645452154453145549425547 543564542415854425543547541543954546452154845145543452 451456524475534354154542549542458145534534542457541459 345564854415524574542415435354254415546541453546425547 954415548435245453415564425345145549425453154254754548

PLAN\#23Q 501585015724.340

589726647918945678647398567894679538246879658791564789 867492735698945678168974576985486917478962567893465789 798456768419259678368794567983687594467892657859417689 689475679485296378176894695875378469426789651897468957 736958847496678592987645187936956487912678495768957648 
Acknowledgements This work has been funded by the Ministry of Economy and Competitiveness of the Government of Spain through project OPTHEUS (ref. PGC2018-095080-B-I00), including European Regional Development Funds (ERDF).

Funding Open Access funding provided thanks to the CRUE-CSIC agreement with Springer Nature.

Open Access This article is licensed under a Creative Commons Attribution 4.0 International License, which permits use, sharing, adaptation, distribution and reproduction in any medium or format, as long as you give appropriate credit to the original author(s) and the source, provide a link to the Creative Commons licence, and indicate if changes were made. The images or other third party material in this article are included in the article's Creative Commons licence, unless indicated otherwise in a credit line to the material. If material is not included in the article's Creative Commons licence and your intended use is not permitted by statutory regulation or exceeds the permitted use, you will need to obtain permission directly from the copyright holder. To view a copy of this licence, visit http://creativecommons.org/licenses/by/4.0/.

\section{References}

1. Aggoune, R.: Minimizing the makespan for the flow shop scheduling problem with availability constraints. Eur. J. Oper. Res. 153(3), 534-543 (2004). https://doi.org/10.1016/S0377-2217(03) 00261-3

2. Bautista, J., Alfaro, R.: A GRASP algorithm for Quota sequences with minimum work overload and forced interruption of operations in a mixed product assembly line. Prog. Artif. Intell. 7, 197-211 (2018). https://doi.org/10.1007/s13748-018-0144-x

3. Bautista, J., Alfaro, R.: Transformación de una línea de montaje de modelos mixtos en un taller de flujo regular: caso de estudio en la factoría Nissan de Barcelona. Dirección y Organización. 69, 99-110 (2019). https://doi.org/10.37610/dyo.v0i69.562

4. Bautista, J., Cano, A., Companys, R., Ribas, I.: Solving the FmlblocklCmax problem using bounded dynamic programming. Eng. Appl. Artif. Intell. 25(6), 1235-1245 (2012). https://doi.org/ 10.1016/j.engappai.2011.09.001

5. Bautista, J., Companys, R., Corominas, A.: Heuristics and exact algorithms for solving the Monden problem. Eur. J. Oper. Res. 88(1), 495-513 (1996). https://doi.org/10.1016/0377-2217(94) 00165-0

6. Bautista-Valhondo, J., Alfaro-Pozo, R.: An expert system to minimize operational costs in mixed-model sequencing problems with activity factor. Expert Syst. Appl. 104, 185-201 (2018). https:// doi.org/10.1016/j.eswa.2018.03.031

7. Bautista-Valhondo, J., Alfaro-Pozo, R.: Mixed integer linear programming models for flow shop scheduling with a demand plan of job types. CEJOR 28, 5-23 (2020). https://doi.org/10.1007/ s10100-018-0553-8

8. Caraffa, V., Ianes, S., Bagchi, T.P., Sriskandarajah, C.: Minimizing makespan in a blocking flowshop using genetic algorithms. Int. J. Prod. Econ. 70(2), 101-115 (2001). https://doi.org/10.1016/ S0925-5273(99)00104-8
9. Choi, K., Kim, C., Kim, H.J.: Multi-Period efficiency and productivity changes in global Automobile: A VRS-VRM and SML productivity index approach. Expert Syst. Appl. 86, 77-86 (2017). https://doi.org/10.1016/j.eswa.2017.05.022

10. Gmys, J., Mezmaz, M., Melab, N., Tuyttens, D.: A computationally efficient branch-and-bound algorithm for the permutation flow-shop scheduling problem. Eur. J. Oper. Res. 284(3), 814-833 (2020). https://doi.org/10.1016/j.ejor.2020.01.039

11. Graham, R.L., Lawler, E.L., Lenstra, J.K., Rinnooy Kan, A.H.G.: Optimization and approximation in deterministic sequencing and scheduling: a survey. Ann. Discrete Math. 5, 287-326 (1979). https://doi.org/10.1016/S0167-5060(08)70356-X

12. Hüttmeir, A., De Treville, S., Van Ackere, A., Monnier, L., Prenninger, J.: Trading off between heijunka and just-in-sequence. Int. J. Prod. Econ. 118(2), 501-507 (2009). https://doi.org/10.1016/j. ijpe.2008.12.014

13. Lei, D., Guo, X.: A parallel neighborhood search for order acceptance and scheduling in flow shop environment. Int. J. Prod. Econ. 165, 12-18 (2015). https://doi.org/10.1016/j.ijpe.2015.03.013

14. Matzka, J., Di Mascolo, M., Furmans, K.: Buffer sizing of a Heijunka Kanban system. J. Intell. Manuf. 23, 49-60 (2012). https:// doi.org/10.1007/s10845-009-0317-3

15. Miltenburg, J.: Level schedules for mixed-model assembly lines in just-in-time production systems. Manage. Sci. 35(2), 192-207 (1989). https://doi.org/10.1287/mnsc.35.2.192

16. Miyata, H.H., Nagano, M.S.: The blocking flow shop scheduling problem: a comprehensive and conceptual review. Expert Syst. Appl. 137, 130-156 (2019). https://doi.org/10.1016/j.eswa.2019. 06.069

17. Monden, Y.: Toyota production system: an integrated approach to just-in-time, 4th edn. Productivity Press, New York (2011)

18. Ozolins, A.: Improved bounded dynamic programming algorithm for solving the blocking flow shop problem. CEJOR (2017). https://doi.org/10.1007/s10100-017-0488-5

19. Pinedo, M.L.: Scheduling (theory, algorithms, and systems). Springer International Publishing, Berlin (2016). https://doi.org/ 10.1007/978-3-319-26580-3

20. Reeves, C.R.: A genetic algorithm for flowshop sequencing. Comput. Oper. Res. 22(1), 5-13 (1995). https://doi.org/10.1016/03050548(93)E0014-K

21. Ronconi, D.P.: A note on constructive heuristics for the flowshop problem with blocking. Int. J. Prod. Econ. 87(1), 39-48 (2004). https://doi.org/10.1016/S0925-5273(03)00065-3

22. Taillard, E.: Some efficient heuristic methods for the flow shop sequencing problem. Eur. J. Oper. Res. 47(1), 65-74 (1990). https://doi.org/10.1016/0377-2217(90)90090-X

23. Yagmahan, B., Yenisey, M.M.: A multi-objective ant colony system algorithm for flow shop scheduling problem. Expert Syst. Appl. 37(2), 1361-1368 (2010). https://doi.org/10.1016/j.eswa. 2009.06.105

Publisher's Note Springer Nature remains neutral with regard to jurisdictional claims in published maps and institutional affiliations. 